\title{
Purification of the mammalian NgBR/hCIT cis-prenyltransferase complex: Identification of a conserved carboxyterminal RxG motif crucial for enzymatic activity
}

\author{
Kariona A. Grabińska, Ban H. Edani, Eon Joo Park, Jan R. Kraehling and William C. Sessa*
}

Department of Pharmacology and Vascular Biology and Therapeutics Program (VBT), Yale University School of Medicine, New Haven, CT 06520, USA

*To whom correspondence should be addressed: William C. Sessa, Ph.D., Vascular Biology \& Therapeutics Program, Department of Pharmacology, Yale University School of Medicine, Amistad Research Building, 10 Amistad St, New Haven, CT 06520, USA, Tel: (203)737-2291; Fax: (203)737- 2290; E-mail: william.sessa@yale.edu 


\section{Summary}

cis-Prenyltransferases (cisPTs) constitute a large family of enzymes conserved during evolution and present in all domains of life. In eukaryotes and archaea, cisPT is the first enzyme committed to the synthesis of dolichyl-phosphate (DolP). DolP is obligate lipid carrier in protein glycosylation reactions in mammals. The homodimeric bacterial enzyme, undecaprenyl diphosphate synthase (UPPS) generates 11 isoprene units and has been structurally and mechanistically characterized in great detail. Recently our group discovered that unlike UPPS, mammalian cisPT is a heteromer consisting of NgBR (NUS1) and hCIT (DHDDS) subunits and this composition has been confirmed in plants and fungal cisPTs. Here, we establish the first purification system for heteromeric cisPT and show that both NgBR and hCIT subunits function in catalysis and substrate binding. Finally, we identified a critical RxG sequence in the Cterminal tail of $\mathrm{NgBR}$ that is conserved and essential for enzyme activity across phyla.

Keywords: polyprenol, dolichol, cis-prenyltransferase, lipid, enzyme, rubber synthesis 


\section{INTRODUCTION}

Dolichyl phosphate is an obligate lipid carrier for protein $\mathrm{N}$-glycosylation, O-mannosylation, Cmannosylation and GPI-anchor synthesis in eukaryotic cells and undecaprenyl phosphate is essential for peptidoglycan biosynthesis in bacteria. cis-Prenyltransferase (cisPT) is the rate limiting enzyme committed to dolichyl phosphate biosynthesis in Eukaryotes, and Archaea as well as undecaprenyl phosphate biosynthesis in Eubacteria $(1,2)$. Both eukaryotic and prokaryotic cisPTs belong to a large protein family, well conserved during evolution (1) and the cisPT family was identified amongst the 355 protein families that trace to the last universal common ancestor of all cells (LUCA, or the progenote) by phylogenetic criteria (3).

The bacterial enzyme, undecaprenyl diphosphate synthase (UPPS) is a homodimeric enzyme that catalyzes chain elongation of farnesyl diphosphate (FPP) by sequential reactions with eight isopentenyl diphosphate molecules (IPP). UPPS has been structurally and mechanistically characterized in great detail $(4,5)$ however, the eukaryotic enzyme has not been purified to date. Our group discovered that unlike UPPS, mammalian and fungal cisPT is heteromeric complex consisting of NgBR (Nus1) and hCIT (DHDDS) subunits in human cells and these findings were confirmed for a number of plant cisPTs (6-10) demonstrating a major difference in the composition of cisPT activity in prokaryotes and eukaryotes. Moreover, loss of function mutations identified in patients via exome sequencing in either $\mathrm{NgBR}$ or hCIT causes a congenital glycosylation disorder, and results in severe clinical manifestations including cognitive defects and retinitis pigmentosa (11-15) and microdeletions within $\mathrm{NgBR}$ locus are linked to pediatric epilepsy(16,17). Further phylogenetic analysis of $\mathrm{NgBR}$ and UPPS suggests 
that a C-terminal motif important for mammalian and fungal cisPT is shared by the single subunit cisPT UPPS (1).

In the present study, we have purified and characterized for the first time a heteromeric cisPT complex composed of NgBR and hCIT subunits. Biochemical characterization of purified wildtype (WT) and various mutants of the $\mathrm{NgBR} / \mathrm{hCIT}$ complex shows that both subunits contribute to catalytic activity. Furthermore, we provide evidence that a conserved C-terminal motif -RxGmotif is critical for enzyme catalysis in both two-component and single-subunit cisPTs. 


\section{RESULTS}

\section{Purification and biochemical characterization of human cis-PT.}

Although NgBR and hCIT or its orthologs are essential subunits for cisPT activity in mammals, fungi and plants, the heteromeric complex and its activity have not been isolated and purified. Thus, we developed a purification scheme to isolate the $\mathrm{NgBR} / \mathrm{hCIT}$ complex (hcisPT). Optimal expression and tagging strategy was first established using a previously described expression system in a triple knockout strain lacking nus $1 \Delta$, rer2 $\Delta$, srt1 $\Delta$ in $S$. cerevisiae (13). Cell survival was ensured due to expression of GlcisPT on the URA3 plasmid and cells were co-transformed with the LEU2 and MET15 plasmids bearing WT or epitope tagged versions of hCIT and $\mathrm{NgBR}$ respectively. The yeast cells were streaked onto complete plates or synthetic complete medium containing 1\% 5-fluoroorotic acid (FOA). Since Ura3 protein, which is expressed from the URA3 marker present in the plasmids, converts FOA to toxic 5-fluorouracil, the survival of the yeast cells on the FOA plate depends on the functionality of the expressed $\mathrm{NgBR} / \mathrm{hCIT}$ complex. hCIT isoform 1 (UniProtKB\# Q86SQ9-1) and isoform 2, (UniProtKB\# Q86SQ9-2), but not isoform 3 (UniProtKB\# Q86SQ9-3) supported the growth of yeast when co-expressed with NgBR in the triple delete strain (data not shown). N- terminal tagging, but not C-terminal tagging of hCIT, generated a construct indistinguishable from wild-type (WT), non-tagged hCIT in yeast. Both Nand $\mathrm{C}$ - terminal tagging of $\mathrm{NgBR}$ reduced its stability and activity when expressed in yeast, therefore a 6-His tag was placed internally after $\mathrm{Gly}^{31}$, between the putative signal anchor and TM1 (Figure 1A). This tag did not affect the growth phenotype and cisPT activity in yeast coexpressing N-terminally Strep-tagged hCIT (not shown). 
Next, the cDNAs encoding 6xHis-NgBR and Strep-tagged-hCIT (Fig1A) were cloned into an IRES-containing bicistronic vector allowing the simultaneous expression of two proteins separately but from the same RNA transcript. This was necessary based on our previous work demonstrating that co-translation of both subunits was required for activity in an in vitro translation system (13). To generate protein for purification, the construct was transiently transfected into Expi293F cells and cells collected 72 hours later by centrifugation. The pellet was washed with PBS, and lysed in detergent free buffer (Ex). To pre-fractionate cell extracts prior to affinity chromatography, crude membrane extracts were subjected to ultracentrifugation, and pellet $(\mathrm{P})$ solubilized by Dounce homogenization in the presence with $0.5 \%$ Triton X100, and solubilized protein cleared by a second ultracentrifugation step (SUP). N-terminally Streptagged hCIT and internally tagged 6xHis-NgBR complex was purified using a dual affinity purification scheme (Figure 1B; Table I). Each step of purification was monitored by Western Blotting and measurement of cisPT activity and this scheme enriched the specific activity of the enzyme 1,700 fold over the starting material. As judged by SDS-PAGE and Coomassie staining of each fraction, the hCIT/NgBR complex was approximately 95\% pure (Figure 1C) and the purified heteromeric complex ran as monodispersed peak on size exclusion chromatography (Figure 1D; black line) that tracked with cisPT activity (Figure 1D; dashed line).

The purified hCIT/NgBR complex required $\mathrm{Mg}^{2+}$ ions for its activity consistent with other studies of the bacterial and eukaryotic enzymes (18-24) with maximum activity at $0.5-2 \mathrm{mM}$ $\mathrm{MgCl}_{2}$. (Figure. 2A). The purified enzyme had a broad $\mathrm{pH}$ optimum (Figure 2B) (19) and was highly stable demonstrating linear cisPT activity occurred over $24 \mathrm{hrs}$ at $37^{\circ} \mathrm{C}$ (Figure 2C). To test the influence of lipids on human cisPT activity, Triton X100 was removed from the enzyme 
preparation by buffer exchange and activity was measured upon the addition of Triton X100 or different phospholipids at concentration above their critical micellar concentration. As seen in Figure 2D, phosphatidic acid modestly increased enzyme activity (1.8 fold) whereas other phospholipids had a marked effect in activating of the enzyme. Cardiolipin increased cisPT activity over 7 fold and phosphatidylcholine, phosphatidylethanolamine, phosphatidylinositol and phosphatidylserine increased activity by approximately 8-12 fold suggesting that the lipid environment strongly impacts cisPT activity.

\section{Protein Sequence Alignment of cis-prenyltransferase homology domain bearing proteins.}

Sequential alignment of nonredundant homomeric cisPTs from all three domains of life together with heteromeric orthologs define the position of cisPT homology domain among NgBR

orthologs (Figure 3). Analysis of the architecture of representative cisPT homology domains revealed number of characteristic features for each of the group (single-subunit orthologs, hCIT orthologs and NgBR orthologs) separately or shared between two groups $(1,4,8,25,26)$. Only four of the previously identified five conserved regions (4) are present in all three groups. The NgBR group lacks Region II and has highly degenerate Regions I and III. Among other conserved residues, the NgBR class is missing a highly-conserved stretch of the six amino acid residues involved in FPP binding and catalysis in Region I (boxed region) found in UPPS and hCIT. Regions IV and V, including the predicted dimer interface based on the structural data obtain for the UPPS of M. luteus and E. coli $(27,28)$ are well conserved amongst all three groups. Finally, the NgBR group shares with homomeric enzymes, but not with the hCIT group, an -RxG C-terminal conserved motif (see red arrowheads)(1,13) with $\mathrm{G}$ being absolutely conserved in EcUPPS and NgBR class. The R residue is substituted with $\mathrm{N}$ in SaUPPS and a majority of plant 
and fungal $\mathrm{NgBR}$ orthologs $(6-10,13,29)$. In a majority of the cases, $\mathrm{X}$ is any nonpolar amino acid however putative Hypocreales NgBR orthologs as well as UPPS of some Trypanosomatidae have positively charged $\mathrm{H}$ or $\mathrm{R}$ at this position. Meta-analysis of available experimental data concerning cisPTs, suggest that both the catalytic motif of Region I and the C-terminal -RxGmotif are indispensable for enzymatic activity being part of the same subunit or separated between hCIT (catalytic motif) and NgBR (RxG) class of cisPTs proteins.

\section{Phenotypic analysis of hcisPT mutants in yeast and biochemical characterization of purified proteins supports importance of RxG motif and position of cisPT homology}

domain in NgBR. To examine the role of the conserved RxG motif in hcisPT, and to test the predicted position of the cis PT homology domain in $\mathrm{NgBR}$, we performed phenotypic analysis of a series of truncation and point mutants of $\mathrm{NgBR}$ or hCIT using the above mentioned yeast triple deletion strain $\operatorname{rer} 2 \Delta, \operatorname{srt} 1 \Delta$, and nus $1 \Delta(13)$. No growth defects were observed with complexes containing $\mathrm{NgBR}^{\mathrm{H} 100 \mathrm{~A}}$ ( $\mathrm{H}$ is the first amino acid in Region I and is highly conserved among proteins bearing cis PT homology domain), $\mathrm{NgBR}^{\mathrm{R} 290 \mathrm{H}}$ (a human mutation causing a glycosylation disorder), $\Delta 85-\mathrm{NgBR}$ (truncated protein lacking first 84 amino acid including TM domain conserved across eukaryotic orthologs of $\mathrm{NgBR}$ ) or $\mathrm{hCIT}^{\mathrm{K} 42 \mathrm{E}}$ (a human mutation causing retinitis pigmentosa) in comparison to WT cisPT composed of $\mathrm{NgBR} / \mathrm{hCIT}$ (Figure 4A). Essentiality of the conserved $\mathrm{D}^{34}$ in hCIT corresponding the $\mathrm{D}^{26}(18,27,30)$ of EcUPPS was confirmed since the hCIT ${ }^{\mathrm{D} 34 \mathrm{~A}} / \mathrm{NgBR}$ complex did not support the growth of triple knockout yeast cells. Cells expressing truncated $\Delta 101-\operatorname{NgBR}$ (lacking the first 100 amino acids) had a severe growth defect suggesting that the first conserved region in $\mathrm{NgBR}$, despite its degeneration, was important for function. This finding is in line with the recent structural analysis of plant $Z, Z$ - 
farnesyl diphosphate synthase (zFPPS) suggesting that N-terminus might be able to extend to the active site of the neighboring monomer near the C-terminus as an important functional regulator (31).

Finally, conservation of the RxG motif was confirmed by the series of mutants leading to severe growth defects. Deletion of the C-terminal $\mathrm{K}^{293}\left(\mathrm{NgBR}^{292}\right)$, the addition of A to $\mathrm{NgBR}$ $\left(\mathrm{NgBR}+\mathrm{A}^{294}\right)$, HA tagging $(\mathrm{NgBR}-\mathrm{HA})$ or $\mathrm{NgBR}^{\mathrm{G} 292 \mathrm{~A}}$ substitutions all reduced growth documenting the importance of the last 4 residues in NgBR for function (Figure 4A). Next, we compared the steady state activities of purified mutants able to support the growth of yeast to the purified WT enzyme. We did not include the N-terminal truncation variants of NgBR since introducing the epitope tag after the predicted TM1 (35-56aa) rendered the enzyme inactive in yeast. As shown in Figure 4B, all mutants analyzed displayed lower enzymatic activity compared to WT enzyme and mutations that strongly impacted growth in yeast $\left(\mathrm{NgBR}^{\mathrm{G} 292 \mathrm{~A}}\right.$, $\mathrm{NgBR} 292$ and $\mathrm{NgBR}+\mathrm{A}^{294}$ ) had the lowest enzymatic activity varying co-substrates FPP and IPP (Figure 4- supplemental figure 1). Next, the polyprenol reaction products were examined by TLC. Since the mutants had reduced activity, no products were detected on the TLC plate when equal amounts of reaction products were loaded from WT enzyme compared to the mutants, therefore an overload of products was used to examine the chain length of prenols generated from the different enzymes. As seen in Figure 4C, WT $\mathrm{NgBR}$ and $\mathrm{NgBR}^{\mathrm{H} 100 \mathrm{~A}}$ generated dominant prenols of $\mathrm{C}_{95}$ (19 units), disease mutants, hCIT $^{\mathrm{K} 42 \mathrm{E}}$ and $\mathrm{NgBR}^{\mathrm{R} 290 \mathrm{H}}$ complexes generated prenols of $\mathrm{C}_{90}$ (18 units) while $\mathrm{NgBR}^{\mathrm{G} 292 \mathrm{~A}}$ and $\mathrm{NgBR}+\mathrm{A}^{294}$ yielded polyprenols that were one or two units shorter. This data support the important role of C-terminus, in particular $\mathrm{G}^{292}$ in cisPT activity. 
To further characterize hcisPT and elucidate the impact of mutations on catalysis and substrate binding, steady state kinetic parameters were measured for complexes containing the WT subunits, hCIT/NgBR, hCIT ${ }^{\mathrm{K} 42 \mathrm{E} / \mathrm{NgBR}, \mathrm{hCIT}_{\mathrm{NgBR}}}{ }^{\mathrm{H} 100 \mathrm{~A}}, \mathrm{hCIT}_{\mathrm{NgBR}}{ }^{\mathrm{R} 290 \mathrm{H}}$ and hCIT/NgBR ${ }^{\mathrm{G} 292 \mathrm{~A}}$ (Table 2). The K42E substitution of hCIT reduced $\mathrm{k}_{\text {cat }}$, and increased the $\mathrm{K}_{\mathrm{m}}$ for FPP but not the affinity for IPP. $\mathrm{NgBR}^{\mathrm{H} 100 \mathrm{~A}}$ and $\mathrm{NgBR}^{\mathrm{R} 290 \mathrm{H}}$ decreased the $\mathrm{k}_{\text {cat }}$ around 4-fold compared to WT enzyme. However, $\mathrm{NgBR}^{\mathrm{H} 100 \mathrm{~A}}$ did not exhibit marked changes in affinity for IPP or FPP, whereas $\mathrm{NgBR}^{\mathrm{R} 290 \mathrm{H}}$ had moderately but significantly reduced affinities for IPP. Substitution of G292A in NgBR, which markedly reduced the growth phenotype in yeast, decreased the catalytic activity of the enzyme to an even greater extent with $\mathrm{k}_{\mathrm{cat}}$ that is 10 -fold lower than the WT. In this case, there were modest (5-fold) but significant differences in the $\mathrm{K}_{\mathrm{m}}$ for IPP, and a slight decrease in the $\mathrm{K}_{\mathrm{m}}$ for FPP.

\section{The RxG motif in homomeric EcUPPS and GIcisPT is critical for cisPT function and enzymatic}

activity. Comparison of the primary amino acid sequences of single- and two-component enzymes reveals that both classes share a conserved RxG C-terminal motif (see Figure 3). Based on the crystal structure of UPPS, $\mathrm{R}^{242}$ in the RXG motif is involved in the binding of the diphosphate group of IPP (30). Further, the role of $\mathrm{C}$ terminus in IPP binding and catalysis is supported by structural information obtained for decaprenyl diphosphate synthase of M. tuberculosis and recent studies on zFPPS of Solanum habrochaites $(31,32)$ implicating the $\mathrm{C}$ terminus in IPP binding. To verify importance of $\mathrm{RxG}$ motif in homomeric cisPTs experimentally, we assayed growth in the triple deletion strain in yeast, transformed with WT or mutant forms of homomeric forms of cisPT, namely EcUPPS and GlcisPT. Cells were transformed with LEU2 plasmids bearing: WT, R242H and G244A of EcUPPS or WT, 
$R 236 H$, and G238A of GlcisPT and phenotypes scored over 7 days. As seen in Figure 5A, WT E.coli and G.lamblia cisPTs exhibited normal growth phenotypes on YPD and FOA plates and R to H mutants for each construct had similar phenotypes compared to WT transformed cells. In contrast, UPPS ${ }^{\mathrm{G} 244 \mathrm{~A}}$ and GlcisPT ${ }^{\mathrm{G} 238 \mathrm{~A}}$ mutants failed to grow on FOA plates demonstrating an essential role of $\mathrm{G}$ in the RxG motif in homomeric cisPT function. To biochemically support the phenotypic data, cisPT activity was measured using purified WT, R236H and G238A mutants of GlcisPT. Mutation of both residues reduced activity, with GlcisPT ${ }^{\mathrm{R} 236 \mathrm{H}}$ having approximately 5 times lower activity and GlcisPT ${ }^{\mathrm{G} 238 \mathrm{~A}}$ being virtually inactive. To verify whether the G238A mutant is enzymatically inactive or generates shorter polyprenols an overload of products was used to examine the chain lengths of undecaprenol from the different enzymes by TLC. As seen in Figure 5C, WT enzyme generated undecaprenyl ( $\mathrm{C}_{55}$ or 11 IPP units), GlcisPT ${ }^{\mathrm{R} 236 \mathrm{H}}$ yielded polyprenols that were one unit shorter compared to WT enzyme ( $\mathrm{C}_{55}$ or 11 IPP units) and no product was observed for GlcisPT ${ }^{\mathrm{G} 238 \mathrm{~A}}$, in agreement with its undetectable enzymatic activity and the phenotypic data.

\section{Presence of heteromeric cisPT in Methanosarcina acetovorans supports the importance of RxG}

motif. Heteromeric cisPTs was predicted in subgroup of Euryarchaeota, based on the fact that Halomebacteria and Archaeoglobaceae have at least two UPPS orthologs: a putative cisPT closely related to single subunit Archea enzymes, but lacking C-terminal RxG motif, and a NgBR/Nus1 like protein $(1,33)$ (Fig. 3). To test the conservation of this motif, we cloned putative undecaprenyl diphosphate synthase of $M$. acetovorans consisting of MaUPPS-A (MA3723, hCIT group) and MaUPPS-B (MA4402, NgBR/Nus1 group). The triple deletion strain bearing GlcisPT on the URA3 plasmid was co-transformed with the LEU2 and MET15 plasmids expressing wild or mutated variants of MaUPPS-A and MaUPPS-B respectively. The growth of yeast cells on the FOA plates was monitored 
over 5 days. As seen in Figure 6A, co-expression of MaUPPS-A and MaUPPS-A is indispensable to support cell growth and neither, MaUPPS-A or MaUPPS-B alone are sufficient. Similarly, as it was observed in case of $\mathrm{NgBR}$, mutation of $\mathrm{His}^{29}$ (corresponding to His ${ }^{100}$ of $\mathrm{NgBR}$ ) and $\mathrm{Arg}^{221}$ (corresponding to $\mathrm{Arg}^{290}$ of NgBR) in MaUPPS-B does not impair growth, but MaUPPS-B ${ }^{\mathrm{G} 223 \mathrm{~A}}$ substitution caused a severe growth delay. Quantification of the cisPT activity reveals that each of the analyzed mutation inhibits enzyme activity with most profound effect of MaUPPS-B ${ }^{\text {G223A }}$ substitution. In accordance with the data on both homomeric and heteromeric enzymes, mutation of the RxG motif negatively influences the chain length of the final prenol product (Figure 6C). 


\section{DISCUSSION}

Here we demonstrate that two subunits of the human cisPT, NgBR and hCIT, are required to form a functional enzyme. The purification of the cisPT complex supports prior work showing that co-translation of both subunits is required for polyprenol synthesis in vitro and for survival in yeast lacking orthologs of each component(13). Moreover, the catalytic $\mathrm{D}^{34}$ in hCIT and RxG motif in NgBR, both conserved in homomeric cisPT such as UPPS, are critical for catalytic activity of the complex. These data highlight the evolutionary conservation of essential elements required for cisPT function throughout all walks of life.

Eukaryotic cisPTs were initially presumed to be homomeric based on detailed studies in undecaprenyl diphosphate synthases of E. coli and M. luteus (4,5,34-36). Recent work by us $(13,25,26)$ and others have shown the essential role of $\mathrm{NgBR}$ (and its orthologs including Nus1 in S. cerevisiae) and hCIT (and its orthologs including Rer2 and Srt1 in S. cerevisiae) as both being required for cisPT activity and polyprenol synthesis $(6,8-10,13)$. The expression of only hCIT or only $\mathrm{NgBR}$ does not support growth in the nus $1 \Delta$, rer $2 \Delta, \operatorname{srt} 1 \Delta$ strain of $S$. cerevisiae and in vitro translation of either subunit is not catalytically active (13). At first glance, this may appear to be in conflict with previous reports showing that eukaryotic hCIT orthologs heterologously expressed in S. cerevisiae were active without co-expression of $\mathrm{NgBR} / \mathrm{Nus} 1$ orthologs. However, this can be explained due to the presence of the endogenous Nus1 in $S$. cerevisiae (7,10,33-35,37-40). Furthermore, a number of hCIT orthologs in plants and protists that were studied in yeast are single-subunit enzymes that have been acquired through horizontal gene transfer, and are more closely related to EcUPPS than to hCIT $(1,41)$. This complexity can be overcome and simplified by complementation experiments in the nus $1 \Delta, \operatorname{rer} 2 \Delta, \operatorname{srt} 1 \Delta$ strain 
as performed previously $(9,13)$ where it is clear that both $\mathrm{NgBR}$ and hCIT or a single-subunit enzyme is required.

Previous experiments have shown that co-expression of $\mathrm{NgBR}$ with hCIT results in their interaction and stabilization based on co-immunoprecipitation experiments $(13,26)$. Whether $\mathrm{NgBR} / \mathrm{Nus} 1$ serves as an accessory subunit for docking the complex to the ER membrane or is a structural component contributing to catalysis and substrate binding was not known. This idea of $\mathrm{NgBR}$ being a docking component was recently reported by Yamashita and co-workers (6) who identified a three-component system composed of HRBP (a NgBR ortholog), HRT (a hCIT ortholog) and rubber elongation factor (42) all of which were required for long chain prenol or natural rubber synthesis when reconstituted into washed rubber particles. Sequential alignment of nonredundant proteins bearing cisPT homology domains presented in this paper enabled the observation of a highly conserved C-terminal RxG motif shared between $\mathrm{NgBR} / \mathrm{Nus} 1$ and the UPPS group of enzymes. Furthermore, meta-analysis of available experimental data concerning cisPT suggests that the $\mathrm{C}$-terminal $\mathrm{RxG}$ motif is indispensable for enzymatic activity. The importance of the $\mathrm{RxG}$ motif is strengthened by the fact that $\mathrm{NgBR}^{\mathrm{R} 290 \mathrm{H}}$ mutation causes a congenital disorder of glycosylation due to defects in dolichol synthesis (13). To further elucidate the role of $\mathrm{NgBR}$ in hcisPT activity, we successfully purified the WT NgBR/hCIT complex and its mutated versions to homogeneity and biochemically characterized the proteins. Mutations in either subunit of hcisPT reduces enzymatic activity in comparison to the WT enzyme and these results are in agreement with the predicted function of the analyzed residue in homomeric cisPTs. 
The first observation supporting a functional role of the extreme C-terminus of NgBR regulating enzyme activity is derived from patients harboring an $\mathrm{R} 290 \mathrm{H}$ mutation in $\mathrm{NgBR}$ which reduces activity in vivo and in vitro (13) and now seen with the purified complex (Table 2) by $70-80 \%$. In addition, epitope tagging the $\mathrm{C}$-terminus of $\mathrm{NgBR}$ reduces activity (Figure 4 ) and this has been previously shown in the CPT-like (CPTL) NgBR ortholog of L. sativa (8). Crystallographic data obtained for homomeric EcUPPS, shows that $\mathrm{Arg}^{242}$ in the RxG motif is involved in $\mathrm{Mg}^{+2}$ IPP binding (30). In addition, the role of the $C$ terminus in IPP binding and catalysis is supported by structural information obtained for decaprenyl diphosphate synthase (DPS) of $M$. tuberculosis (32) and zFPPS(31). In the structure of DPS, the extreme C terminus of one monomer of decaprenyl diphosphate synthase interacts with the active site of the other subunit. Based on our biochemical data and the available structural information for homomeric cisPTs, it is likely that $\mathrm{Arg}^{290}$ in the RxG motif of NgBR is involved in IPP binding and the flexible nature of glycine in the motif, may permit conformational changes to seal the active site cavity. Collectively these data imply that both subunits contribute to enzymatic activity and the C-terminal tail of $\mathrm{NgBR}$ regulates aspects of cisPT catalytic activity.

The general function of $\mathrm{NgBR}$ orthologs as a cisPT subunit is further supported by the heteromeric cisPT from M. acetovorans. MaUPPS-B (NgBR ortholog) is missing any predicted TM domains and yet MaUPPS-A/ MaUPPS-B complex is indispensable for supporting the growth of triple deletion strain of $S$. cerevisiae. Since the mutations in conserved region of MaUPPS-B have similar impact on MaUPPS activity as those in distantly related hcisPT, we postulate a common mechanism for both enzymes. 
In summary, this study strongly advances the concept that eukaryotic cisPT is composed of two subunits, $\mathrm{NgBR}$ and hCIT, and this two component system is conserved in

Methanosarcina acetovorans. Interestingly, conservation of the $\mathrm{RxG}$ motif across phyla implicates a catalytic role of $\mathrm{NgBR}$ orthologs in the synthesis of polyprenol diphosphates critical for cellular function. Future structural studies on the heteromeric NgBR/hCIT complex will permit a deeper mechanistic understanding of how the $\mathrm{C}$ terminus regulates substrate binding and catalysis. 


\section{Materials}

Unless otherwise stated, all reagents were of analytical grade and purchased from Sigma Aldrich (St. Louis MO), Thermo Fisher Scientific (Waltham, MA), Zymo Research (Irvine, CA). Restriction enzymes were from New England Biolabs (Ipswich, MA). [1- $\left.{ }^{14} \mathrm{C}\right]-\mathrm{IPP}(50$ mCi/mmol) was purchased from American Radiolabeled Chemicals (St. Luis, MO). Reverse phase thin layer chromatography (RP18-HTLC) plates were from Merck (Darmstad, Germany). Dolichol 19 standards was purchased from American Radiolabeled Chemicals (St. Luis, MO) and undecaprenol was obtained from Isoprenoids Collection of Institute of Biochemistry and Biophysics, PAS. Primary antibodies used in this study include $\alpha$-NgBR (Abcam, ab168351), $\alpha$ DHDDS (Sigma, SAB2100572), $\alpha$-Strep-tag (IBA Solutions for Life Sciences, StrepMABClassic). Site-directed mutagenesis was performed using TagMaster Site-Directed Mutagenesis Kit (GM Biosciences). The Escherichia coli UPPS was amplified from genomic DNA. Methanosarcina acetovorans ORFs encoding MaUPPS-A (MA3723) and MaUPPS-B (MA4402) were amplified form synthetically synthetized codon optimized for expression in $S$. cerevisiae gBlocks ${ }^{\circledR}$ Gene Fragments (ITD integrated Technologies, Coralville, Iowa). GlcisPT, $\mathrm{NgBR}$ and hCIT isoform 1 were amplified from previous plasmids (REF). The cassette containing synthetic intron and internal ribosome entry site (IRES) was amplified form pIRESneo1 (Clontech). Constructs used in this study are listed in Table I-S. cloning primers are listed in Table II-S and mutagenesis primers are listed III-S. Invitrogen Gateway Cloning strategy was used to insert cDNA into yeast expression vectors. All PCR products were cloned into pCR8/GW/TOPO TA (Invitrogen), sequenced and sub-cloned into final yeast expression vector pKG-GW1 or pKG-GW2. To express His-Strep tagII- GlcisPT in bacteria, the Strep tagIIGlcisPT was amplified with restriction enzyme recognition sites and Strep -tag sequence inserted 
in the primers. The PCR product was ligated in frame with internal 6 His tag of pRSF-DUET1 plasmid.

To express both subunits of hcisPT from single mRNA in mammalian cells, the internal ribosome entry site (IRES) surrounded by two multicloning sites was introduced into pCEP4 (Invitrogen) using NEBUilder HiFi DNA Assembly (NEB) to obtain the pKGmDUET vector (details Supplemental Method). 6-His internally tagged NgBR was sub-cloned into BamH I/Not I sites of pKGmDUET from pKG-GW2-(31-HIS)NgBR plasmid and SmaI/EcoRI/ Klenow treated Strep-hCIT was sub-cloned into PmeI site from pKG-GW-Strep-hCIT plasmid.

\section{Yeast complementation assay.}

For yeast complementation analysis of cisPTs, S. cerevisiae strains KG405 (nus14 rer2A srt14), carrying the GlcisPT gene on a plasmid with a URA3 marker was used (43). To analyze homomeric GlcisPT and EcUPPS mutants, strain KG405 was transformed with vector pKG-GW1 (leucine selection) carrying WT or mutated versions of corresponding genes, or empty vector as negative control. To phenotypically analyze hcisPT, strain KG405 was transformed with vectors $\mathrm{pKG-GW1}$ carrying hCIT variants (leucine selection) and pKGGW2 carrying NgBR variants (methionine selection) in combination, or empty vectors as negative control. To analyze putative heteromeric UPPS of Methanosarcina acetovorans (MaUPPS), strain KG405 was transformed with vector $\mathrm{pKG-GW1} \mathrm{carrying} \mathrm{MaUPPS-A}$ (MA3723, hCIT ortholog) and vector pKG-GW2 carrying WT and mutated variants of MaUPPS-B (MA4402, NgBR ortholog) in combination or with the corresponding empty vectors as negative control. 
Transformed yeast cells were grown overnight at $30^{\circ} \mathrm{C}$ in synthetic defined medium lacking uracil and leucine or uracil, methionine and leucine were streaked onto synthetic defined medium containing all amino acids, nucleotide supplements and 1\% (w/v) 5-FOA (Zymo Research) and onto YPD plates. The plates were incubated for up to 7 days at $30{ }^{\circ} \mathrm{C}$. Colonies growing on the 5-FOA plates were streaked on synthetic defined medium lacking uracil and incubated at $30^{\circ} \mathrm{C}$ for 3 days to verify the loss of the pNEV-GlcisPT plasmid. Yeast strain KG405 and its derivative carrying MaUPPS complex, hcisPT complex or single subunits enzymes expressed from pKG-GW1 plasmid instead of pNEV-GlcisPT were cultured in $2 \%(\mathrm{wt} / \mathrm{vol})$ Bacto peptone and $1 \%(\mathrm{wt} / \mathrm{vol})$ yeast extract supplemented with $2 \%$ glucose (wt/vol) (YPD). Synthetic minimal media were made of $0.67 \%(\mathrm{wt} / \mathrm{vol})$ yeast nitrogen base and $2 \%$ (wt/vol) supplemented with auxotrophic requirements. For solid media, agar (BD, Sparks MD) was added at a $2 \%(\mathrm{wt} / \mathrm{vol})$ final concentration. Yeast cells were transformed using the Frozen-EZ Yeast Transformation II Kit (Zymo Research).

\section{Purification of human cisPT.}

To purify the human hcisPT complex, constructs containing internally tagged $6 \mathrm{His}-\mathrm{NgBR}$ and N-terminally tagged Strep-hCIT(pKGmDUET-hCIT/NgBR) were transiently transfected in 200 $\mathrm{ml}$ culture of Expi293F cells according to the manufacturer's protocol (Invitrogen). Cells were harvested $72 \mathrm{hr}$ post post-transfection by centrifugation, and washed with PBS. Each gram of the cells was re-suspended in S-tactin buffer (100 mM Tris HCL, pH 8, 300 mM NaCl, 2 mM 2mercaptoethanol, $1 \mathrm{mM} \mathrm{MgCl} 2$ ) and cells were disrupted by sonication on ice. Unbroken material was cleared with a $1000 \mathrm{~g}$ centrifugation, and the supernatant from this spin was recentrifuged at $200000 \mathrm{~g}$ for $30 \mathrm{~min}$ at $4^{\circ} \mathrm{C}$ to obtain total membrane fractions. Total membrane 
fraction was homogenized in Stactin buffer supplemented with 0.5\% Triton X100 to solubilize membrane proteins. Solubilization was followed by additional 200,000 x g centrifugation. StrephCIT was purified from 200,000 g supernatant using the Strep-Tactin XT system (IBA GmbH). Strep-hCIT/6-His-NgBR complex was eluted from Strep-Tactin XT resin with $100 \mathrm{mM}$ Tris HCL, pH 8, 300 mM NaCl, 2 mM 2-mercaptoethanol, 1 mM $\mathrm{MgCl}_{2}, 0.1 \%$ Triton X 100, 5\% glycerol, $50 \mathrm{mM}$ biotin elution buffer. The Strep-Tactin purification of Strep-hCIT was followed by nickel column purification of 6His-NgBR using HisPur Ni-NTA resin (Thermo Fisher Scientific ). Sephadex G-25 in PD-10 Desalting Columns (GE Healthcare Life Sciences) were used to exchange buffer for $20 \mathrm{mM} \mathrm{NaPi}$, pH 8, $300 \mathrm{mM} \mathrm{NaCl}, 2 \mathrm{mM}$ 2-mercaptoethanol, $1 \mathrm{mM}$ $\mathrm{MgCl}_{2}, 0.1 \%$ Triton X 100, 20\% glycerol. Purification efficiency was tracked by Western blot analysis of each fraction, Coomassie Staining of SDS PAGE of final eluate and measurement of specific cisPT activity (Figure 1 and Table 1).

\section{Purification of GlcisPT.}

To purify GlcisPT, pRSF-DUET1- GlcisPT plasmid was transformed into E. coli Rosetta 2 cells (Novagen). E. coli was grow in auto-induction medium (44) till the logarithmic growth phase at $37^{\circ} \mathrm{C}$ flowed $24 \mathrm{hr}$ incubation at $17^{\circ} \mathrm{C}$ to express heterologous protein. Cells were harvested by centrifugation, washed with PBS and stored $-80^{\circ} \mathrm{C}$. The proteins were extracted using B-PER reagent (Thermo Fisher Scientific ) supplemented with $1 \mathrm{mM} \mathrm{MgCl}_{2}$ and $2 \mathrm{mM} 2$ mercaptoethanol. The GlcisPT was first purified using nickel column HisPur Ni-NTA resin (Thermo Fisher Scientific) followed by the Strep-Tactin XF (IBA GmbH) purification. Sephadex G-25 in PD-10 Desalting Columns (GE Healthcare Life Sciences) were used to exchange buffer for $20 \mathrm{mM} \mathrm{NaPi}$, pH 8, $300 \mathrm{mM} \mathrm{NaCl}, 2 \mathrm{mM}$ 2-mercaptoethanol, $1 \mathrm{mM} \mathrm{MgCl} 2,0.1 \%$ Triton X 
100, 20\% glycerol. Purification efficiency was tracked by Western blot analysis and Coomassie Staining of SDS PAGE of final eluate.

\section{Size Exclusion Chromatography}

The size exclusion chromatography was carried out using an AKTA $^{\mathrm{TM}}$ purifier (GE Healthcare Life Sciences, Pittsburgh, PA, USA) with the size exclusion column Superdex ${ }^{\text {TM }} 200$ 10/300 GL (GE Healthcare Life Sciences) at flow rate of $0.5 \mathrm{ml} / \mathrm{min}$. The UV absorption at $254 \mathrm{~nm}$ was measured as a readout for protein elution. The used buffer contained $50 \mathrm{mM}$ TrisHCl buffer, $\mathrm{pH}$ 8.0 and $300 \mathrm{mM} \mathrm{NaCl}, 2 \mathrm{mM}$ 2-mercaptoethanol, $1 \mathrm{mM} \mathrm{MgCl} 2,0.1 \%$ Triton X100, 5 \% (v/v) glycerol. The column was calibrated by using the LMW- and HMW-calibration kit (GE Healthcare Life Sciences). For determination of the void volume Blue Dextran (2,000 kDA; GE Healthcare Life Sciences) was used. Size determination was calculated based on the standard linear equation based on the calibration of the column.

\section{cisPT enzymatic activity of hcisPT}

Standard incubation mixture contained, in a final volume of $50 \mu 1,50 \mu \mathrm{M}$ FPP, $100 \mu \mathrm{M}$ [1- 14C]IPP (55 mCi/mmol) 50 mM Tris- $\mathrm{HCl}$ pH 8, 1 mM MgCI $2,20 \mathrm{mM}$ 2-mercaptoethanol, 1mg/ml BSA, 10mM KF and $1 \%(\mathrm{w} / \mathrm{v})$ phosphatidylinositol. Membrane or crude protein $(100 \mu \mathrm{g})$ or 20$100 \mathrm{ng}$ of purified protein was used for activity assays. In some experiments with crude membranes, zaragozic acid A $(10 \mu \mathrm{M})$ was added. Reactions were incubated for $60 \mathrm{~min}$ at $37^{\circ} \mathrm{C}$ and terminated by the addition of $1 \mathrm{ml}$ of chloroform-methanol 3:2. The protein pellet was removed by centrifugation and the supernatant was washed three times with 1/5 volume of 10 mM EDTA in $0.9 \% \mathrm{NaCl}$. The incorporation of ${ }^{14} \mathrm{C}$-IPP into organic fractions containing 
polyprenyl diphosphate was measured by scintillation counting. To analyze the length of polyprenol diphosphates lipids were subjected to mild acid hydrolysis $\left(1 \mathrm{hr}\right.$ incubation at $80{ }^{\circ} \mathrm{C}$ in $500 \mu \mathrm{l}$ of $1 \mathrm{~N}$ HCL solution). Dephosphorylated lipids were extracted 3 times with 1 volume of hexane. Organic fraction was washed with 1 volume of water. Then organic solvent was evaporated and lipids were loaded together with dolichol 19 as internal standard onto HPTLC RP-18 plates with and run in acetone containing $50 \mathrm{mM} \mathrm{H}_{3} \mathrm{P}_{4}$. Plates were exposed to film to visualize the products of IPP incorporation.

\section{Kinetics parameters}

Standard $25 \mu \mathrm{l}$ or $100 \mu \mathrm{l}\left(\mathrm{hCIT} / \mathrm{NgBR}^{\mathrm{G} 292}\right.$ mutant) reaction mixture containing 9-25nM hcisPT was used. To measure kinetic parameters for FPP, 0.1-50 $\mu \mathrm{M}$ FPP was use along with $100 \mu \mathrm{M}$ IPP for wild type enzyme, $\mathrm{hCIT}^{\mathrm{K} 42 \mathrm{E}}$ and $\mathrm{NgBR}^{\mathrm{H} 100 \mathrm{~A}}$ mutants, $200 \mu \mathrm{M}$ IPP for $\mathrm{NgBR}^{\mathrm{R} 292 \mathrm{H}}$ mutant and $400 \mu \mathrm{M}$ IPP for $\mathrm{NgBR}^{\mathrm{G} 292}$ mutant. To measure kinetic parameters for IPP, $0.1-400 \mu \mathrm{M}$ IPP was use along with $50 \mu \mathrm{M}$ FPP. The initial velocity data were fitted to Michaelis-Menten equation using the GraphPad Prism 7.02 computer program (GraphPad Software, Inc.) to obtain $K m$ values. $k_{\text {cat }}$ values were obtained from Michaelis-Menten equation for IPP.

\section{cisPT enzymatic activity of GlcisPT}

GlcisPT activity was measured as before((41) with minor modifications. Briefly the incubation mixture contained, in a final volume of $50 \mu \mathrm{l}, 45 \mu \mathrm{M}$ FPP, $100 \mu \mathrm{M}$ [1- 14C]- IPP $(55 \mathrm{mCi} / \mathrm{mmol})$ 25 mM Tris- $\mathrm{HCl}$ pH 7.4, 1 mM MgCI 2,20 mM 2-mercaptoethanol, 10 mM KF, 0.1\% Triton $\mathrm{X} 100,1 \mathrm{mg} / \mathrm{ml} \mathrm{BSA}$ and $2 \mu \mathrm{g}$ of purified enzyme. After $60 \mathrm{~min}$ incubation at $37^{\circ} \mathrm{C}$, the reaction was terminated by the addition of $1 \mathrm{ml}$ of chloroform-methanol 3:2. The protein pellet was 
removed by centrifugation and the supernatant was washed three times with $1 / 5$ volume of 10 mM EDTA in $0.9 \% \mathrm{NaCl}$. The incorporation of 14C-IPP into organic fractions containing polyprenyl diphosphate was measured by scintillation counting. To analyze the length of polyprenol diphosphates the $200 \mu$, reaction was stopped by addition of HCL to obtain $1 \mathrm{~N}$ final concentration. Lipids were subjected to mild acid hydrolysis, extracted with hexane and loaded onto HPTLC RP-18 together with undecaprenol as internal standard. After running in acetone containing $50 \mathrm{mM} \mathrm{H}_{3} \mathrm{PO}_{4}$ plates were exposed to film to visualize the products of IPP incorporation.

\section{cisPT enzymatic activity of MaUPPS}

For S. cerevisiae expressing MaUPPS complex membrane fractions were prepared as described (45)and cisPT activity measured $(45,46)$ with minor modifications. Briefly the incubation mixture contained, in a final volume of $100 \mu \mathrm{l}, 45 \mu \mathrm{M}$ FPP, $100 \mu \mathrm{M}$ [1- 14C]- IPP (55 mCi/mmol) 25 mM Tris-HCl pH 7.4, 1 mM MgCI 2,20 mM 2-mercaptoethanol, 10 mM KF, 10 $\mu \mathrm{M}$ Zaragozic acid A and $250 \mu \mathrm{g}$ of membranes protein. After $90 \mathrm{~min}$ incubation at $30^{\circ} \mathrm{C}$, the reaction was terminated by the addition of $4 \mathrm{ml}$ of chloroform-methanol 3:2. The protein pellet was removed by centrifugation and the supernatant was washed three times with $1 / 5$ volume of $10 \mathrm{mM}$ EDTA in $0.9 \% \mathrm{NaCl}$. The organic phase was concentrated under a stream of nitrogen. Then organic solvent was evaporated and lipids were loaded onto HPTLC RP-18 precoated plates with a concentrating zone and run in acetone containing $50 \mathrm{mM} \mathrm{H}_{3} \mathrm{PO}_{4}$. Plates were exposed to film to visualize the products of IPP incorporation. To measure incorporation of radioactive IPP into polyprenol fraction, the gel from the zone containing radiolabeled polyprenols was scraped and subjected to liquid scintillation counting. To analyze the length of 
polyprenol diphosphates before running the TLC analysis lipids were subjected to mild acid hydrolysis. Lipids were loaded onto HPTLC RP-18 together with undecaprenol as internal standard and subjected to radio-autography as was described above. 


\section{Acknowledgments}

This work was supported by Grants R01 HL64793, R01 HL61371 and HL133018 from the

National Institutes of Health and the Leducq Fondation (MIRVAD network) to WCS and an American Heart Association Scientist Development Grant to EJP. We acknowledge the gift of undecaprenol from Dr. Ewa Kula-Swiezewska (Polish Academy of Science). 
References:

1. Grabińska, K. A., Park, E. J., and Sessa, W. C. (2016) cis-Prenyltransferase: New Insights into Protein Glycosylation, Rubber Synthesis, and Human Diseases. Journal of Biological Chemistry 291, 18582-18590

2. Hartley, M. D., and Imperiali, B. (2012) At the membrane frontier: a prospectus on the remarkable evolutionary conservation of polyprenols and polyprenyl-phosphates. Archives of biochemistry and biophysics 517, 83-97

3. Weiss, M. C., Sousa, F. L., Mrnjavac, N., Neukirchen, S., Roettger, M., Nelson-Sathi, S., and Martin, W. F. (2016) The physiology and habitat of the last universal common ancestor. Nature Microbiology 1, 16116

4. Takahashi, S., and Koyama, T. (2006) Structure and function of cis-prenyl chain elongating enzymes. The Chemical Record 6, 194-205

5. Teng, K.-H., and Liang, P.-H. (2012) Structures, mechanisms and inhibitors of undecaprenyl diphosphate synthase: a cis-prenyltransferase for bacterial peptidoglycan biosynthesis. Bioorganic chemistry 43, 51-57

6. Yamashita, S., Yamaguchi, H., Waki, T., Aoki, Y., Mizuno, M., Yanbe, F., Ishii, T., Funaki, A., Tozawa, Y., and Miyagi-Inoue, Y. (2016) Identification and reconstitution of the rubber biosynthetic machinery on rubber particles from Hevea brasiliensis. eLife $\mathbf{5}$, e19022

7. Kwon, M., Kwon, E.-J., and Ro, D. (2016) cis-Prenyltransferase and Polymer Analysis from a Natural Rubber Perspective. Methods in Enzymology

8. Qu, Y., Chakrabarty, R., Tran, H. T., Kwon, E.-J. G., Kwon, M., Nguyen, T.-D., and Ro, D.-K. (2015) A lettuce (Lactuca sativa) homolog of human Nogo-B receptor interacts with cis-prenyltransferase and is necessary for natural rubber biosynthesis. Journal of Biological Chemistry 290, 1898-1914

9. Epping, J., van Deenen, N., Niephaus, E., Stolze, A., Fricke, J., Huber, C., Eisenreich, W., Twyman, R. M., Prüfer, D., and Gronover, C. S. (2015) A rubber transferase activator is necessary for natural rubber biosynthesis in dandelion. Nature Plants 1

10. Brasher, M. I., Surmacz, L., Leong, B., Pitcher, J., Swiezewska, E., Pichersky, E., and Akhtar, T. A. (2015) A two-component enzyme complex is required for dolichol biosynthesis in tomato. The Plant Journal 82, 903-914

11. Sabry, S., Vuillaumier-Barrot, S., Mintet, E., Fasseu, M., Valayannopoulos, V., Héron, D., Dorison, N., Mignot, C., Seta, N., and Chantret, I. (2016) A case of fatal Type I congenital disorders of glycosylation (CDG I) associated with low dehydrodolichol diphosphate synthase (DHDDS) activity. Orphanet Journal of Rare Diseases 11, 84

12. Venturini, G., Koskiniemi-Kuendig, H., Harper, S., Berson, E. L., and Rivolta, C. (2014) Two specific mutations are prevalent causes of recessive retinitis pigmentosa in North American patients of Jewish ancestry. Genetics in Medicine 17, 285-290

13. Park, E. J., Grabińska, K. A., Guan, Z., Stránecký, V., Hartmannová, H., Hodaňová, K., Barešová, V., Sovová, J., Jozsef, L., and Ondrušková, N. (2014) Mutation of Nogo-B receptor, a subunit of cis-prenyltransferase, causes a congenital disorder of glycosylation. Cell metabolism 20, 448-457

14. Züchner, S., Dallman, J., Wen, R., Beecham, G., Naj, A., Farooq, A., Kohli, M. A., Whitehead, P. L., Hulme, W., and Konidari, I. (2011) Whole-exome sequencing links a 
variant in DHDDS to retinitis pigmentosa. The American Journal of Human Genetics $\mathbf{8 8}$, 201-206

15. Zelinger, L., Banin, E., Obolensky, A., Mizrahi-Meissonnier, L., Beryozkin, A., BandahRozenfeld, D., Frenkel, S., Ben-Yosef, T., Merin, S., and Schwartz, S. B. (2011) A missense mutation in DHDDS, encoding dehydrodolichyl diphosphate synthase, is associated with autosomal-recessive retinitis pigmentosa in Ashkenazi Jews. The American Journal of Human Genetics 88, 207-215

16. Milani, D., Cagnoli, G. A., Baccarin, M., Alfei, E., Guerneri, S., and Esposito, S. (2016) Insights into 6q21-q22: Refinement of the critical region for acro-cardio-facial syndrome. Congenital anomalies 56, 187-189

17. Szafranski, P., Von Allmen, G. K., Graham, B. H., Wilfong, A. A., Kang, S.-H. L., Ferreira, J. A., Upton, S. J., Moeschler, J. B., Bi, W., and Rosenfeld, J. A. (2015) 6q22. 1 microdeletion and susceptibility to pediatric epilepsy. European Journal of Human Genetics 23, 173-179

18. Guo, R.-T., Ko, T.-P., Chen, A. P.-C., Kuo, C.-J., Wang, A. H.-J., and Liang, P.-H. (2005) Crystal Structures of Undecaprenyl Pyrophosphate Synthase in Complex with Magnesium, Isopentenyl Pyrophosphate, and Farnesyl Thiopyrophosphate ROLES OF THE METAL ION AND CONSERVED RESIDUES IN CATALYSIS. Journal of Biological Chemistry 280, 20762-20774

19. Akira, K., Hiroshi, S., and Kyozo, O. (1996) Proteolytic release of dehydrodolichyl diphosphate synthase from pig testis microsomes. Bioscience, biotechnology, and biochemistry 60, 1109-1114

20. Adair, W. L., and Cafmeyer, N. (1987) Characterization of the Saccharomyces cerevisiae cis-prenyltransferase required for dolichyl phosphate biosynthesis. Archives of biochemistry and biophysics 259, 589-596

21. Adair, W., Cafmeyer, N., and Keller, R. (1984) Solubilization and characterization of the long chain prenyltransferase involved in dolichyl phosphate biosynthesis. Journal of Biological Chemistry 259, 4441-4446

22. Takahashi, I., and Ogura, K. (1982) Prenyltransferases of Bacillus subtilis: undecaprenyl pyrophosphate synthetase and geranylgeranyl pyrophosphate synthetase. The Journal of Biochemistry 92, 1527-1537

23. Oh, S. K., Han, K. H., Ryu, S. B., and Kang, H. (2000) Molecular Cloning, Expression, and Functional Analysis of a cis-Prenyltransferase from Arabidopsis thaliana IMPLICATIONS IN RUBBER BIOSYNTHESIS. Journal of Biological Chemistry 275, 18482-18488

24. Apfel, C. M., Takács, B., Fountoulakis, M., Stieger, M., and Keck, W. (1999) Use of genomics to identify bacterial undecaprenyl pyrophosphate synthetase: cloning, expression, and characterization of the essential uppS gene. Journal of bacteriology 181, 483-492

25. Miao, R. Q., Gao, Y., Harrison, K. D., Prendergast, J., Acevedo, L. M., Yu, J., Hu, F., Strittmatter, S. M., and Sessa, W. C. (2006) Identification of a receptor necessary for Nogo-B stimulated chemotaxis and morphogenesis of endothelial cells. Proceedings of the National Academy of Sciences 103, 10997-11002

26. Harrison, K. D., Park, E. J., Gao, N., Kuo, A., Rush, J. S., Waechter, C. J., Lehrman, M. A., and Sessa, W. C. (2011) Nogo-B receptor is necessary for cellular dolichol biosynthesis and protein N-glycosylation. The EMBO journal 30, 2490-2500 
27. Ko, T.-P., Chen, Y.-K., Robinson, H., Tsai, P.-C., Gao, Y.-G., Chen, A. P.-C., Wang, A. H.-J., and Liang, P.-H. (2001) Mechanism of Product Chain Length Determination and the Role of a Flexible Loop in Escherichia coliUndecaprenyl-pyrophosphate Synthase Catalysis. Journal of Biological Chemistry 276, 47474-47482

28. Fujihashi, M., Zhang, Y.-W., Higuchi, Y., Li, X.-Y., Koyama, T., and Miki, K. (2001) Crystal structure of cis-prenyl chain elongating enzyme, undecaprenyl diphosphate synthase. Proceedings of the National Academy of Sciences 98, 4337-4342

29. Zhang, H., Ohyama, K., Boudet, J., Chen, Z., Yang, J., Zhang, M., Muranaka, T., Maurel, C., Zhu, J.-K., and Gong, Z. (2008) Dolichol biosynthesis and its effects on the unfolded protein response and abiotic stress resistance in Arabidopsis. The Plant Cell 20, 18791898

30. Chang, S.-Y., Ko, T.-P., Liang, P.-H., and Wang, A. H.-J. (2003) Catalytic mechanism revealed by the crystal structure of undecaprenyl pyrophosphate synthase in complex with sulfate, magnesium, and triton. Journal of Biological Chemistry 278, 29298-29307

31. Chan, Y.-T., Ko, T.-P., Yao, S.-H., Chen, Y.-W., Lee, C.-C., and Wang, A. H.-J. (2017) Crystal Structure and Potential Head-to-Middle Condensation Function of a Z, ZFarnesyl Diphosphate Synthase. ACS Omega 2, 930-936

32. Wang, W., Dong, C., McNeil, M., Kaur, D., Mahapatra, S., Crick, D. C., and Naismith, J. H. (2008) The structural basis of chain length control in Rv1086. Journal of molecular biology 381, 129-140

33. Ogawa, T., Emi, K. i., Koga, K., Yoshimura, T., and Hemmi, H. (2016) A cisprenyltransferase from Methanosarcina acetivorans catalyzes both head-to-tail and nonhead-to-tail prenyl condensation. The FEBS journal

34. Shridas, P., Rush, J. S., and Waechter, C. J. (2003) Identification and characterization of a cDNA encoding a long-chain cis-isoprenyltranferase involved in dolichyl monophosphate biosynthesis in the ER of brain cells. Biochemical and biophysical research communications 312, 1349-1356

35. Endo, S., Zhang, Y.-W., Takahashi, S., and Koyama, T. (2003) Identification of human dehydrodolichyl diphosphate synthase gene. Biochimica Et Biophysica Acta (BBA)-Gene Structure and Expression 1625, 291-295

36. Sato, M., Sato, K., Nishikawa, S.-i., Hirata, A., Kato, J.-i., and Nakano, A. (1999) The Yeast RER2 Gene, Identified by Endoplasmic Reticulum Protein Localization Mutations, Encodescis-Prenyltransferase, a Key Enzyme in Dolichol Synthesis. Molecular and cellular biology 19, 471-483

37. Akhtar, T. A., Matsuba, Y., Schauvinhold, I., Yu, G., Lees, H. A., Klein, S. E., and Pichersky, E. (2013) The tomato cis-prenyltransferase gene family. The Plant Journal 73, 640-652

38. Schmidt, T., Lenders, M., Hillebrand, A., van Deenen, N., Munt, O., Reichelt, R., Eisenreich, W., Fischer, R., Prüfer, D., and Gronover, C. S. (2010) Characterization of rubber particles and rubber chain elongation in Taraxacum koksaghyz. BMC biochemistry 11, 11

39. Asawatreratanakul, K., Zhang, Y. W., Wititsuwannakul, D., Wititsuwannakul, R., Takahashi, S., Rattanapittayaporn, A., and Koyama, T. (2003) Molecular cloning, expression and characterization of cDNA encoding cis-prenyltransferases from Hevea brasiliensis. European Journal of Biochemistry 270, 4671-4680 
40. Juchimiuk, M., Orłowski, J., Gawarecka, K., Świeżewska, E., Ernst, J. F., and Palamarczyk, G. (2014) Candida albicans cis-prenyltransferase Rer2 is required for protein glycosylation, cell wall integrity and hypha formation. Fungal Genetics and Biology 69, 1-12

41. Grabińska, K. A., Cui, J., Chatterjee, A., Guan, Z., Raetz, C. R., Robbins, P. W., and Samuelson, J. (2010) Molecular characterization of the cis-prenyltransferase of Giardia lamblia. Glycobiology 20, 824-832

42. Dennis, M., Henzel, W., Bell, J., Kohr, W., and Light, D. (1989) Amino acid sequence of rubber elongation factor protein associated with rubber particles in Hevea latex. Journal of Biological Chemistry 264, 18618-18626

43. Park, E. J., Grabińska, K. A., Guan, Z., and Sessa, W. C. (2016) NgBR is essential for endothelial cell glycosylation and vascular development. EMBO reports 17, 167-177

44. Studier, F. W. (2005) Protein production by auto-induction in high-density shaking cultures. Protein expression and purification 41, 207-234

45. Szkopińska, A., Grabińska, K., Delourme, D., Karst, F., Rytka, J., and Palamarczyk, G. (1997) Polyprenol formation in the yeast Saccharomyces cerevisiae: effect of farnesyl diphosphate synthase overexpression. Journal of lipid research 38, 962-968

46. Grabińska, K., Sosińska, G., Orłowski, J., Swiezewska, E., Berges, T., Karst, F., and Palamarczyk, G. (2005) Functional relationships between the Saccharomyces cerevisiae cis-prenyltransferases required for dolichol biosynthesis. Acta Biochim Pol 52, 221-232 


\section{Figure Legends}

Figure 1. Purification of NgBR/hCIT cis-PT complex. A. Epitope tagged NgBR and hCIT were transiently expressed in Expi293 cells from bicistronic vector and purified as decribed in the text (SA, putative signal anchor; TM, putative transmembrane domain). $\mathbf{B}$ is a representative western blot monitoring purification of NgBR/hCIT complex (Ex-Total Crude Extract, P200,000g membrane pellet, SUP- 200,000g supernatant after Triton X100 solubilization of the membrane fraction, FT1 and FT2- flow through from Strep-Tactin and Nickle column respectively, E1-elution from the Strep-tactin and E2- final elution from the Nickle column). C is a Coomassie stained SDS/PAGE showing SUP, FT1, E1, two (E2a) and eight times concentrated final fraction(E2b). D shows size exclusion chromatography profile of purified $\mathrm{NgBR} / \mathrm{hCIT}$ cisPT complex. UV absorption at $254 \mathrm{~nm}$ was measured as a readout for protein elution (left y axis, black line). Incorporation of $\mathrm{C}^{14}$ labeled IPP into organic fraction was measured as a readout for cis-PT activity (right y axis, red line).

Figure 2. Biochemical properties of purified human cis-PT. cis-PT activity was measured using purified hcis-PT complex as described in Material and Methods unless otherwise stated. A. Optimal concentration of $\mathrm{MgCl}_{2}$. cis-PT activity was measured in the presence of 5mM EDTA (to chelate $\mathrm{MgCl}_{2}$ in the enzyme storage buffer,) or $0.1,0.5,1,2,5,10$ and $50 \mathrm{mM} \mathrm{MgCl}$. B. $\mathrm{pH}$ dependence of cis-PT activity. cis-PT activity was measure in the $50 \mathrm{mM}$ sodium phosphate buffer (pH 5.5; 6.1;7.4; 8.0) or 50 mM Tris $\mathrm{HCl}(\mathrm{pH} 7.5 ; 8.0 ; 8.5 ; 9.0 ; 9.3)$. C. Time dependence of $c i s-P T$ activity. D. Phospholipids stimulate $c i s$-PT activity. cis-PT activity was measured in the buffer containing $0.35 \%$ (w/v) Triton X100, $1 \%$ (w/v) Phosphatidic acid (PA), 1\% (w/v) 
Cardiolipin (CL), 1\% (w/v) Phosphatidylcholine (PC), 1\% (w/v) Phosphatidylethanolamine (PE), $1 \%(w / v)$ Phosphatidylinositol (PI) or $1 \%(w / v)$ Phosphatidylserine. Data are mean \pm S.D values of three technical replicates,

Figure 3. Multiple alignment of the proteins bearing cis-PT homology domain. Proteins represented in the figure are: single subunit cis-PTs: EcUPPS (Escherichia coli, GenBank P60472), MIUPPS (Micrococcus luteus; GeneBank BAA31993.1), SaUPPS (Sulfolobus acidocaldarius; GenBank WP_011277635.1) and GlcisPT (Giardia lamblia; GenBank XP_001709868.1); orthologes of hCIT cis-PT subunit: hCIT (human, GenBank BAB14439), ScRer2 (Saccaraomyces cerevisiae, GenBank P35196), MaUPPS-A (Methanosarcina acetovorans, GenBank AAM07075.1); orthologes of NgBR cis-PT subunit: NgBR (human, GenBank NP_612468), ScNus1 (Saccaraomyces cerevisiae, GenBank NP_010088), MaUPPS-B (Methanosarcina acetovorans, GenBank) WP_011024281.1). The conservation scoring is performed by PRALINE. The scoring scheme works from 0 for the least conserved alignment position, up to $10(*)$ for the most conserved alignment position. Position of five conserved regions originally described among cis-PTs is underlined. Residues involved in catalysis and substrate binding conserved between hCIT orthologues and single subunit cis-PTs are boxed. Red triangles indicate position of conserved Arginine/Asparagine and Glycine in C-terminal RxG- motif of NgBR orthologues and homomeric cis-PTs. Black triangle indicates position of strictly conserved C-terminal Phenylalanine/Tyrosine in cis-PT homology domain.

Figure 4. Characterization of human cis-PT mutants. A. The rer $2 \Delta$, srt $1 \Delta$, nus $1 \Delta$ triple deletion strain expressing $G$. lamblia cis-PT from URA3 plasmid was co-transformed with the 
LEU2 and MET15 plasmids bearing wild or mutated variant of hCIT and NgBR as indicated. The cells were streaked onto complete plates (YPD) or synthetic complete medium containing $1 \%$ 5fluoroorotic acid (FOA). The Ura3 protein, which is expressed from the URA3 marker present in the plasmids, converts FOA to toxic 5-fluorouracil. The growth of cells was monitored overtime to assess phenotypic differences. The combination of alleles affecting the growth is indicated in italic and the combination not supporting the growth is marked in red. B. cis-PT activity was measured using purified WT or mutant hcisPT complex as described in Material and Methods. Mean \pm S.D values of 3-9 independent measurements from at least 2 independent isolations of enzyme except for hCIT/NgBR+A ${ }^{294}$ and $\mathrm{hCIT} / \mathrm{NgBR}^{292}$ performed on one batch.). C. Reverse-phase TLC of dephosphorylated polyprenols formed by hisPT mutants. Equal amounts of product were analyzed to reveal quality differences.

Figure 4 - figure supplement 1. Michaelis-Menten plot of hcisPT kinetics. A The measurements of Km for FPP and B. IPP. Each data points represent 3-8 independent

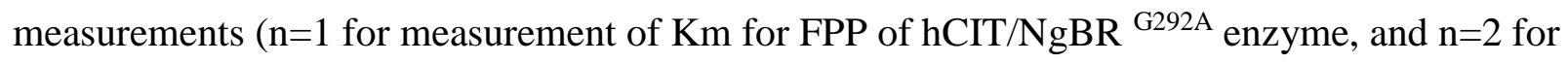
other samples).. Details are provided under "Materials and Methods.”.

\section{Figure. 5. Characterization of EcUPPS and Glcis-PT mutants in RxG motif reveals is}

essentiality. Panel A. The rer $2 \Delta, \operatorname{srt} 1 \Delta$, nus $1 \Delta$ triple deletion strain expressing G. lamblia cisPT from $U R A 3$ plasmid was transformed with the LEU2 plasmid bearing WT or mutants ORFs for GlcisPT or EcUPPS. As a negative control, cells were transformed with empty vector. The mutated genes G244A and G238A did not supporting the growth and are underlined. B. cis-PT 
activity was measured using purified wild type or mutant Glcis-PT. Data are mean \pm S.D values

of three technical replicates. C. Reverse-phase TLC of dephosphorylated polyprenols formed by purified Glcis-PT.

Fig 6. Phenotypical and biochemical analysis of heteromeric MaUPPS supports predicted length of cis-PT domain of NgBR and importance of C-terminal RxG motif. A. The rer2 $\triangle$, srt1A, nus1 1 triple deletion strain expressing G. lamblia cis-PT from URA3 plasmid was cotransformed with the LEU2 and MET15 plasmids bearing wild or mutated variant of MaUPPS-A (hCIT/Rer2/Srt1 orthologue) and MaUPPS-B (NgBR/Nus1 orthologue) as indicated. Cells transformed with empty plasmid were used as negative control and cells transformed with $L E U 2$ GlcisPT or co transformed with hCIT (LEU2) and NgBR (MET15) were used as positive control. The cells were streaked onto YPD or synthetic complete medium containing $1 \% 5$ fluoroorotic acid (FOA). The growth of cells was monitored overtime to assess phenotypic differences. The combination of alleles affecting the growth is indicated in italics and the combination not supporting the growth is underlined B. cis-PT activity was measured using $S$. cerevisiae rer $2 \Delta, \operatorname{srt} 1 \Delta$, nus $1 \Delta$ membrane fractions expressing WT or mutated MaUPPs subunits as indicated. Data are mean \pm S.D values of 4-5 replicates in two independent experiments. . C. Reverse-phase TLC of dephosphorylated polyprenols formed by heteromeric wt or mutaed MaUPPS expressed in $S$. cerevisiae rer2 $\Delta$, srt1 $\Delta$, nus $1 \Delta$ mutants. 
A.

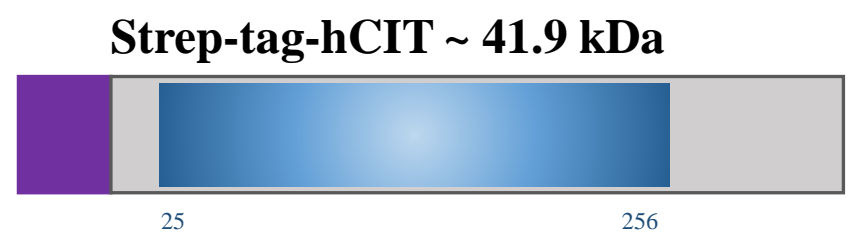

His-tag-NgBR 34.1 kDa

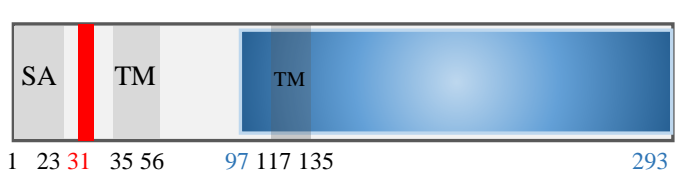

C.
B.
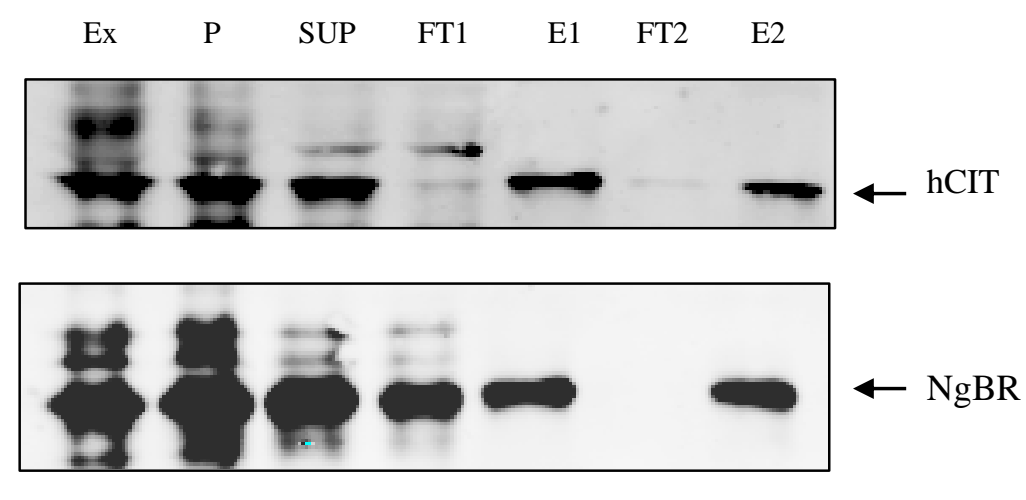

D.

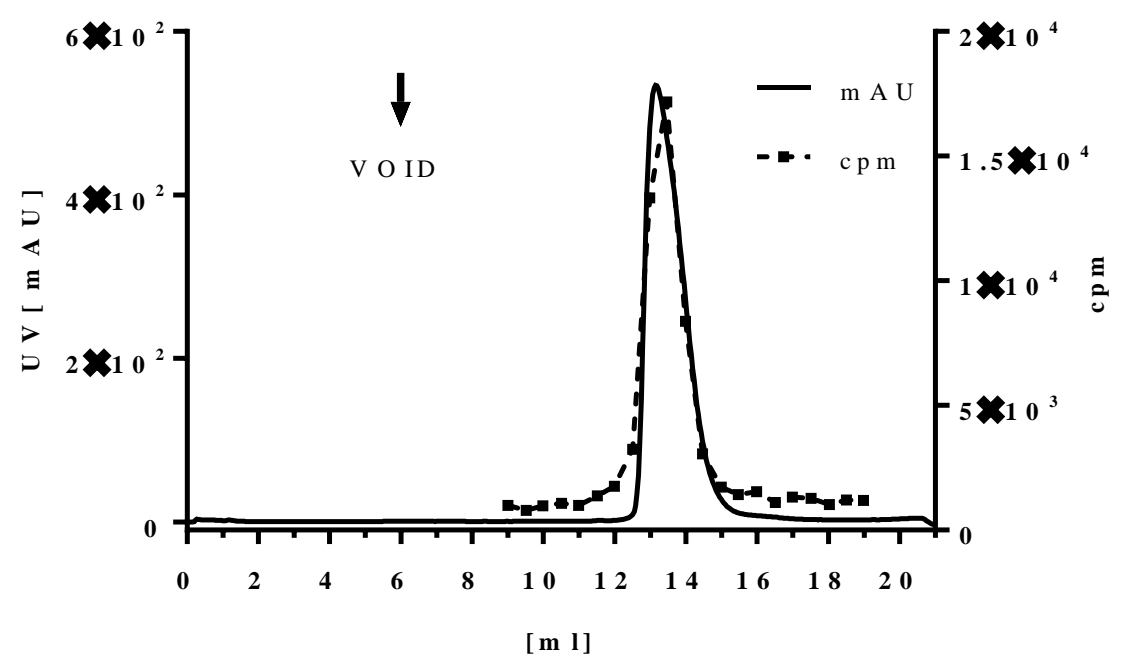

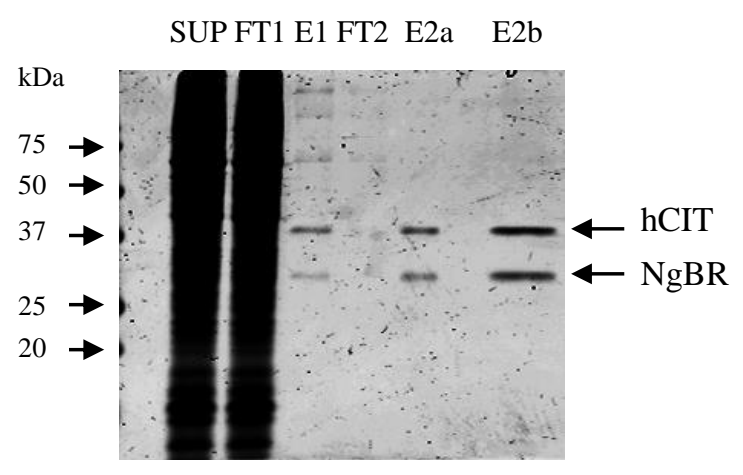


Fig. 2

A.

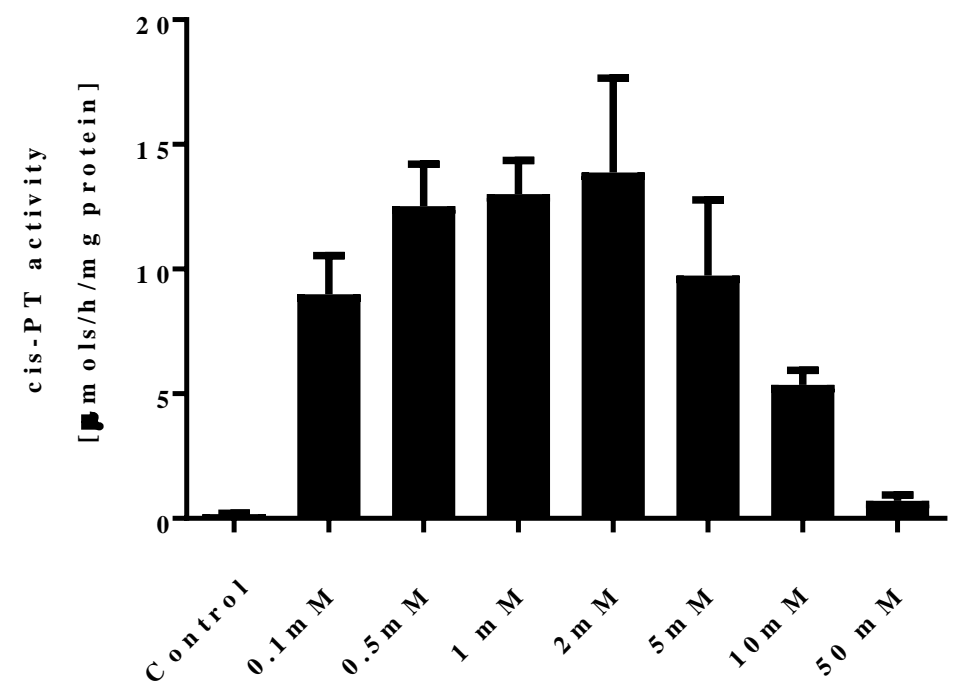

C.

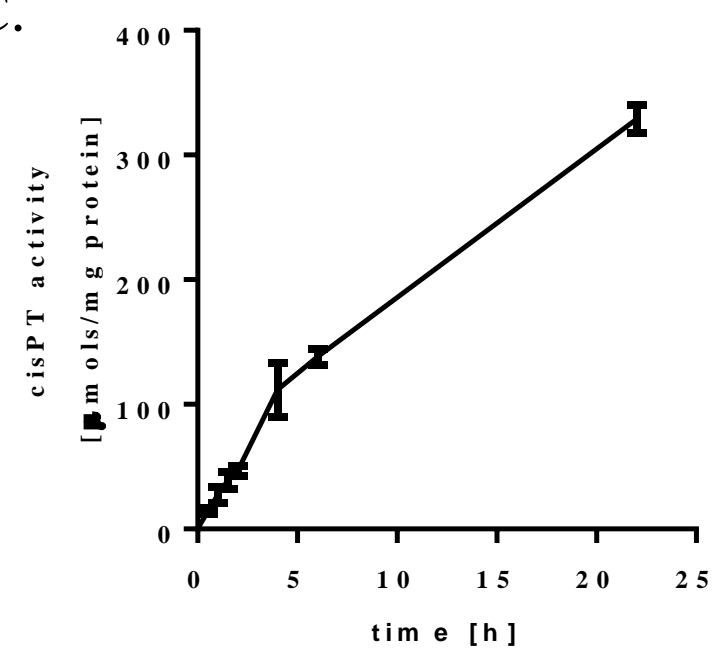

B.

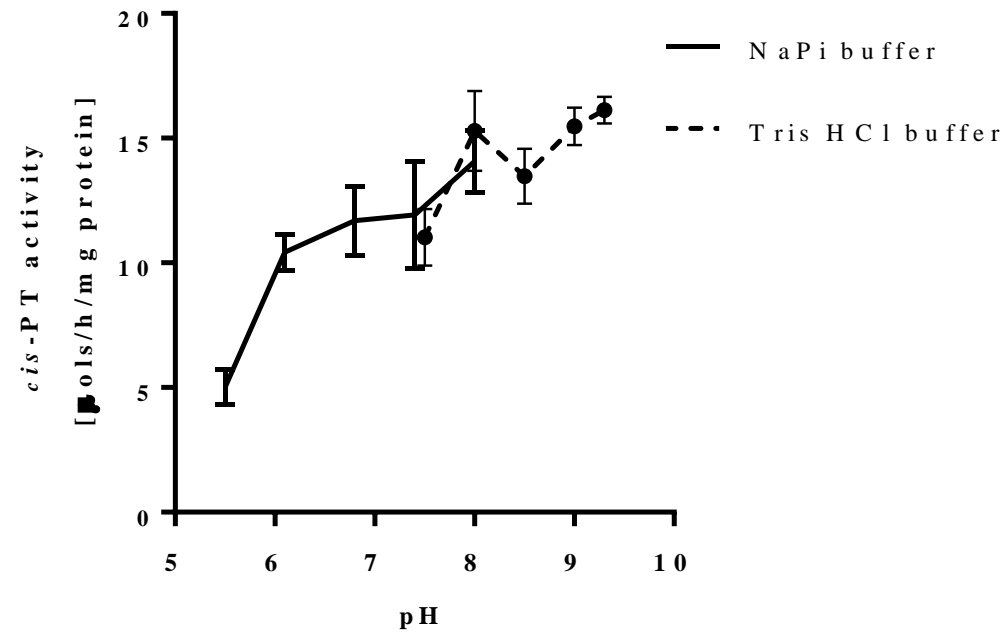

D.

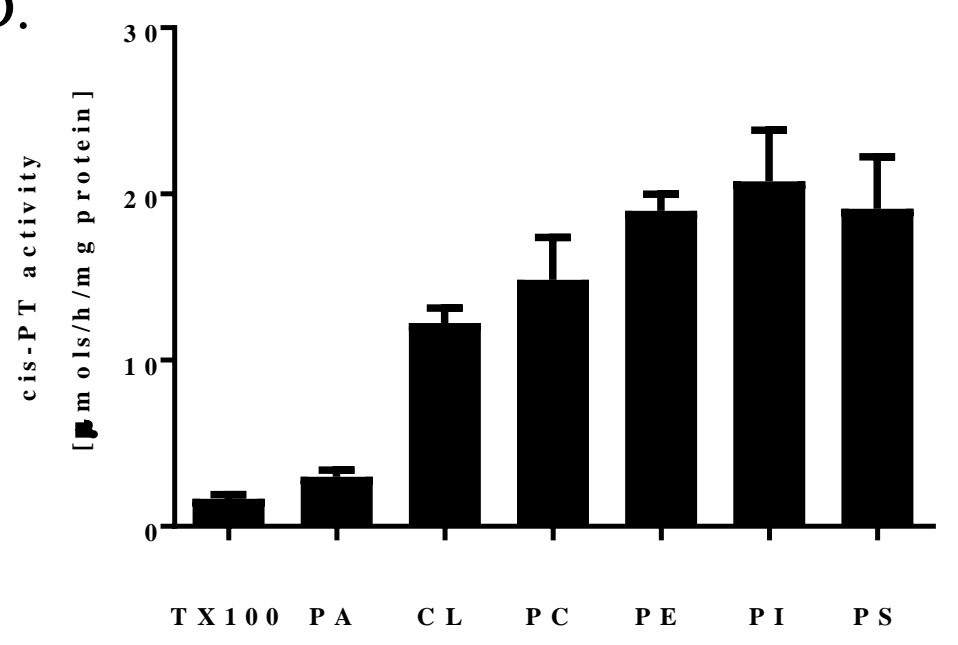


Fig. 3

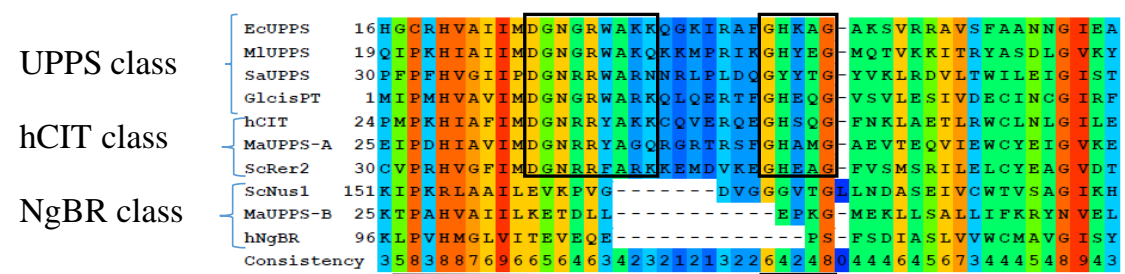

Region I

Region II

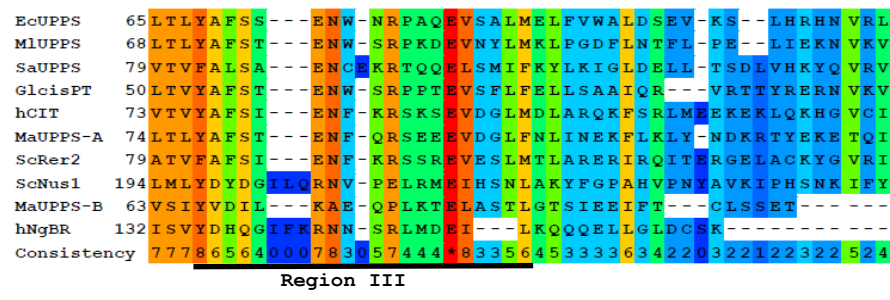

ECUPPS 108RIIGD-TSRFNSRLQERIRKSEALTAGNTGLTLNIAANYGGRDIVQ GVR MIUPPS 111ETIGF-IDDLPDHTRKAVLEAKEKTRHNTGLTIVFALNYGRKEIISAVQ SaUPPS 125KAIGM-LDKLPEDLKKLVVDLESTTEKYNKKRLILAICYGGRQEILDAIR G1CISPT 93 QFCGERTRIPETVIAAMNCIEQDTAACTELISVCFNYGGHEIAQACR MaUPPS-A 119RVIGD-RTRLPAFLNKSIIERTERATETHRKFNLNVAIAYGGRODIMOAVR SCRer2 125 KIIGD-LSILDKSLLEDVRVAVETTRNNRRATLNICFPYTGREETLH AMK SCNUS1 243NID----GIETETDVGNEIEANOERDRIAIEISLLSNRDGRETIVD ITK MaUPPS-B 100 - . - . - - GYKIHGLEGEVRSSRQGRDFFVYVSLGFGGRGEITR AVI hNgBR $167-\ldots$ Consistency $33342022243334343542445465333343847543658837 * 5476$

\begin{tabular}{|c|c|}
\hline EPPS & PDQIDEEMLNQHVCMH- - ELAPVDI \\
\hline & EISETHFNEYIFTA - - NMPDPEIIIR \\
\hline aUPPS & 174 KIMNDYKLGIID \\
\hline ICISPT & 143 SVLADCLEGDAV \\
\hline IT & 168 EMAWGVEQGLLD \\
\hline MaUPPS-A & $168 \mathrm{DIAACVSSGKLS}$ \\
\hline SoRer2 & AIDESTLESHIYTA \\
\hline SoNus1 1 & 289 TMAELCAVNELS \\
\hline MaUPPS-B & 139 S ILEEVKTGSVS \\
\hline hNgBR & $206 \mathrm{DFCQLVAQKQKR}$ \\
\hline Consistenc & 44575654645494436 \\
\hline cUpps & YFTDVLWPDFDEQDFEGALNAFANRE \\
\hline MlUPPS & VFIDEFWPDFNESLAQC \\
\hline SaUPPS & FFVDVYWPDFRKIDIWRAIRSF $Q$ KRK \\
\hline GlCisPT & IFSDLLWPEFSVRAFKDCLDEFASRT \\
\hline hСIT & VFQPVIWPEYTFWNLFEAILQFQMNH \\
\hline MaUPPS-A 2 & YFCAPFWPEFRKIDLLRSIRVYQARK \\
\hline ScRer2 & ELLDCLWPEFGPIRMAWILLKFS FHK \\
\hline SoNus1 & YWERD-NNEVIYSVFIRGLRQYA GCK \\
\hline MaUPPS-B & ELL- YFTDVNWRDVRRIDLLRVIRDFQ KRQ \\
\hline hNgBR & 244 FGPVDSTLGFLPHIRL--TEI--VSIPSHL-NISYEDFFSLRQYA ACE \\
\hline ac & acy $675436776 * 84 *$ \\
\hline & $\bar{n}$ \\
\hline & 241 RRFG \\
\hline MlUPPS & 244 RRFGGI \\
\hline SAUPPS 2 & $258 \mathrm{RNFGA}_{-}$ \\
\hline Glcispt & $235 \mathrm{R}$ \\
\hline $\begin{array}{l}\text { hCIT } \\
\text { MaUPPS-A }\end{array}$ & $\begin{array}{l}252 \mathrm{SVLQKAR} \\
257 \mathrm{DEKKQEN}\end{array}$ \\
\hline ScRer2 & \\
\hline ScNus1 & \\
\hline & \\
\hline & - \\
\hline
\end{tabular}

Consistency 44565 
Fig. 4

A.
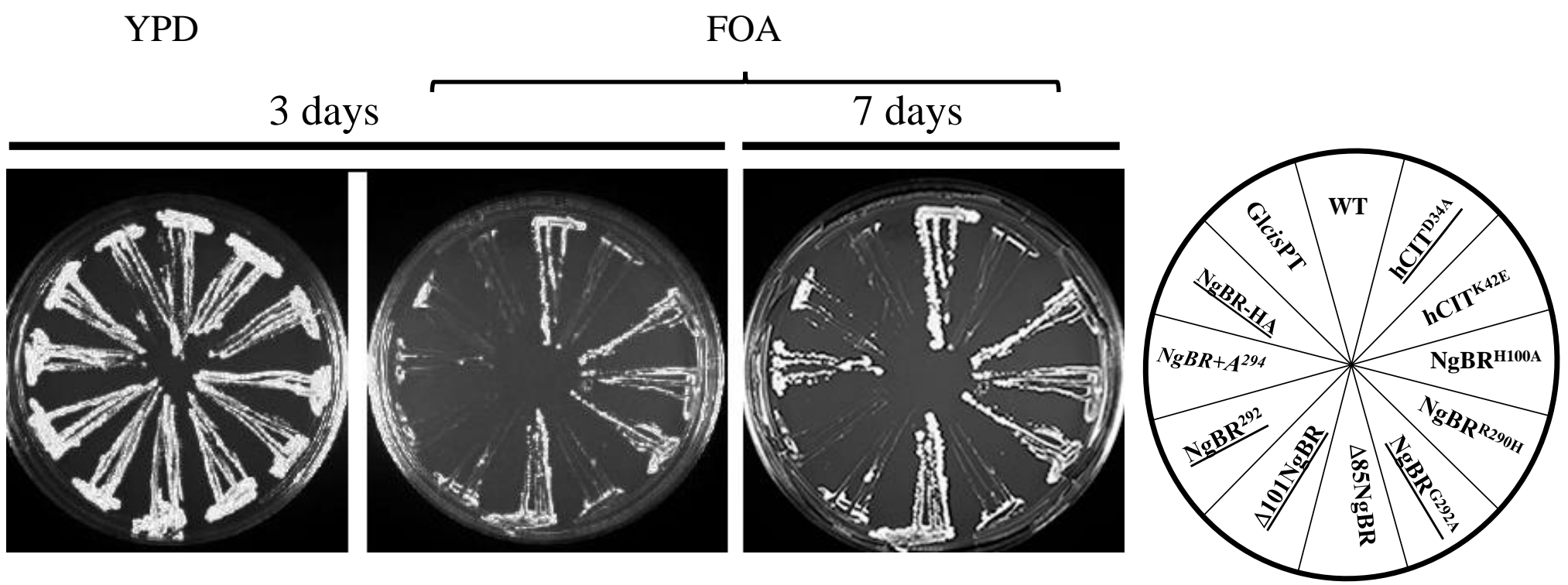

B.

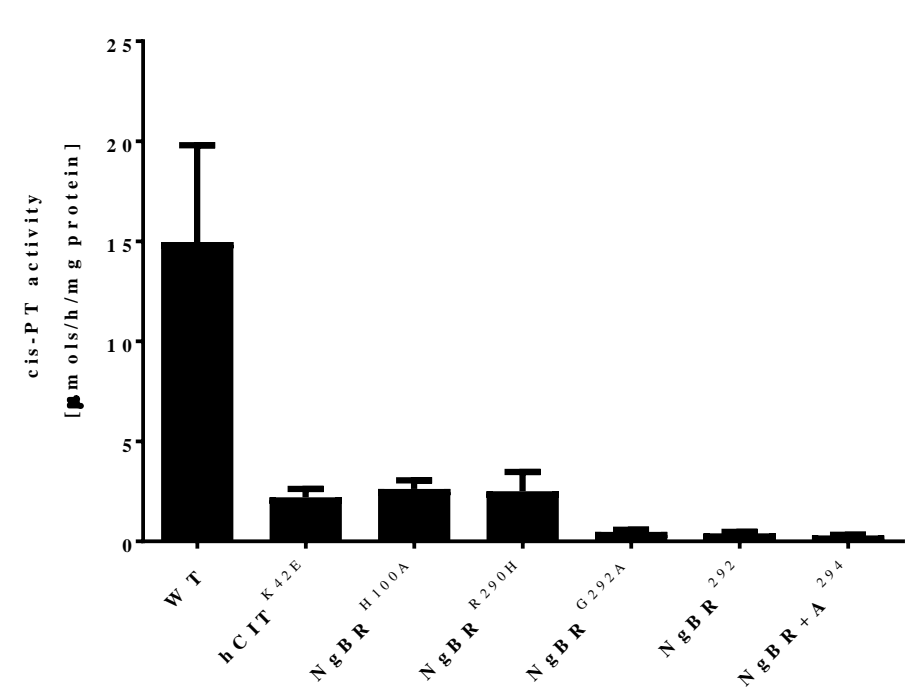

C.

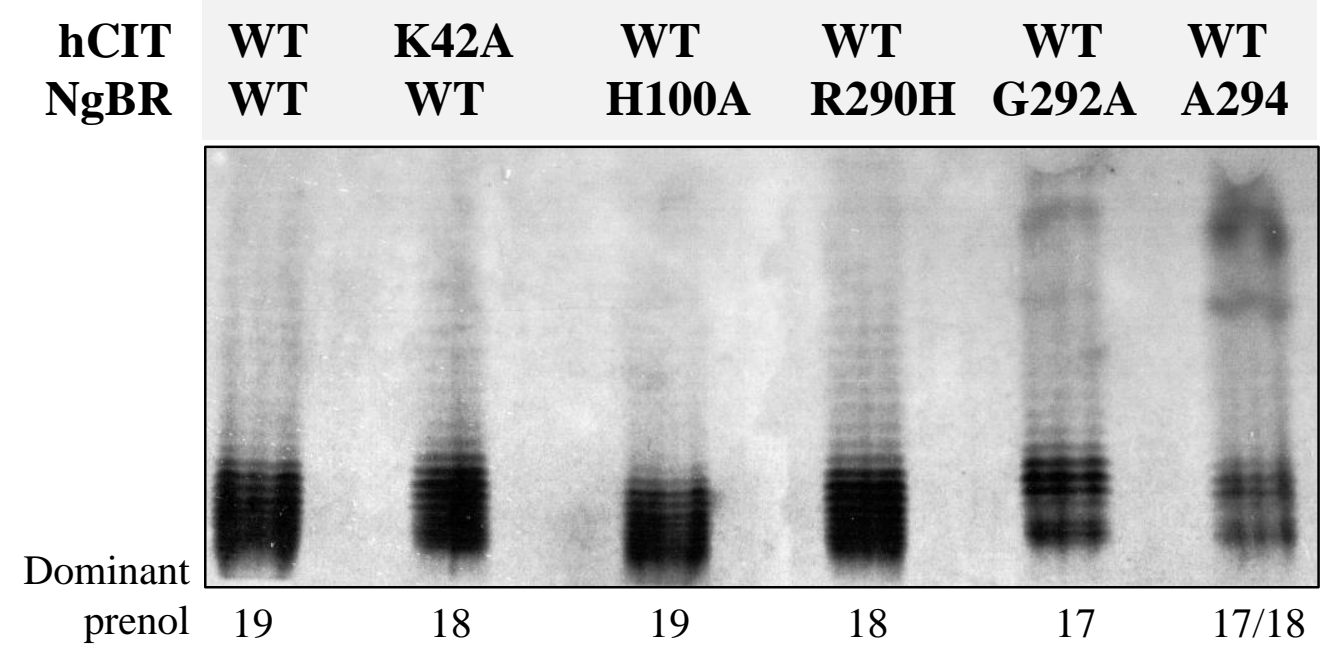




\section{Fig. 4- supplemental fig 1}

A

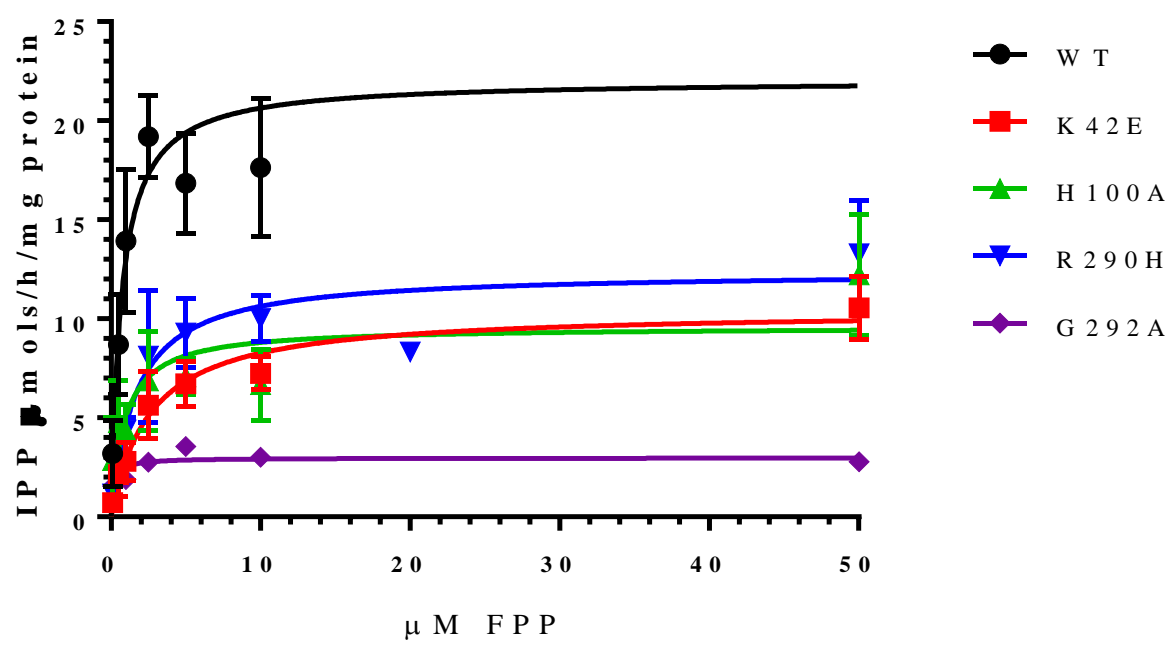

B

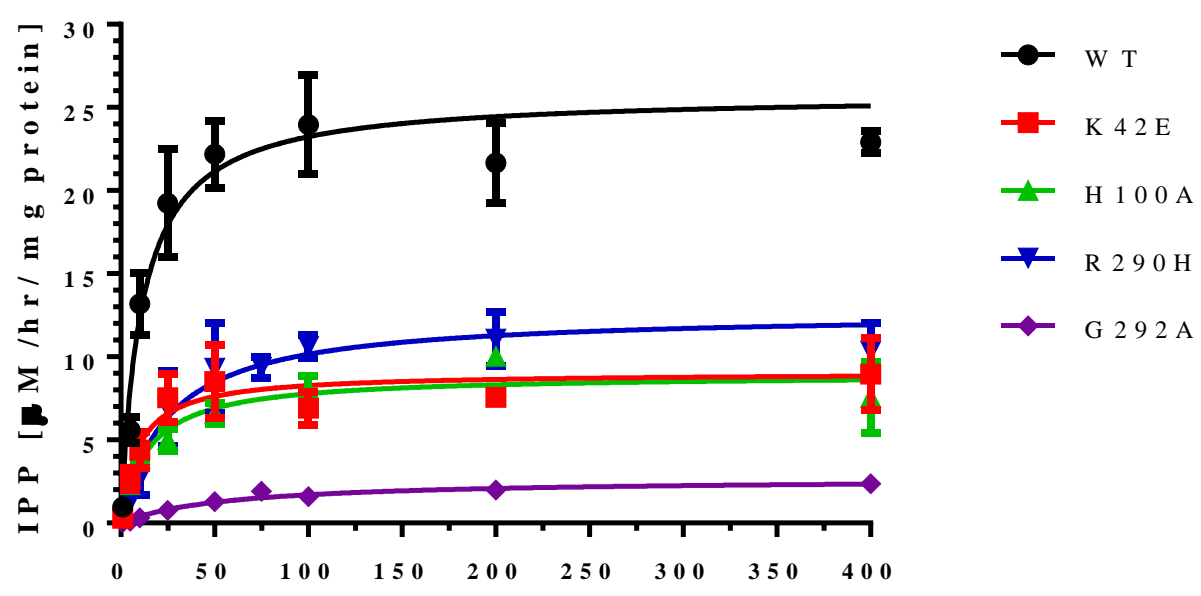

I P P [ $\mu \mathrm{M}]$ 


\section{Fig. 5.}

A.
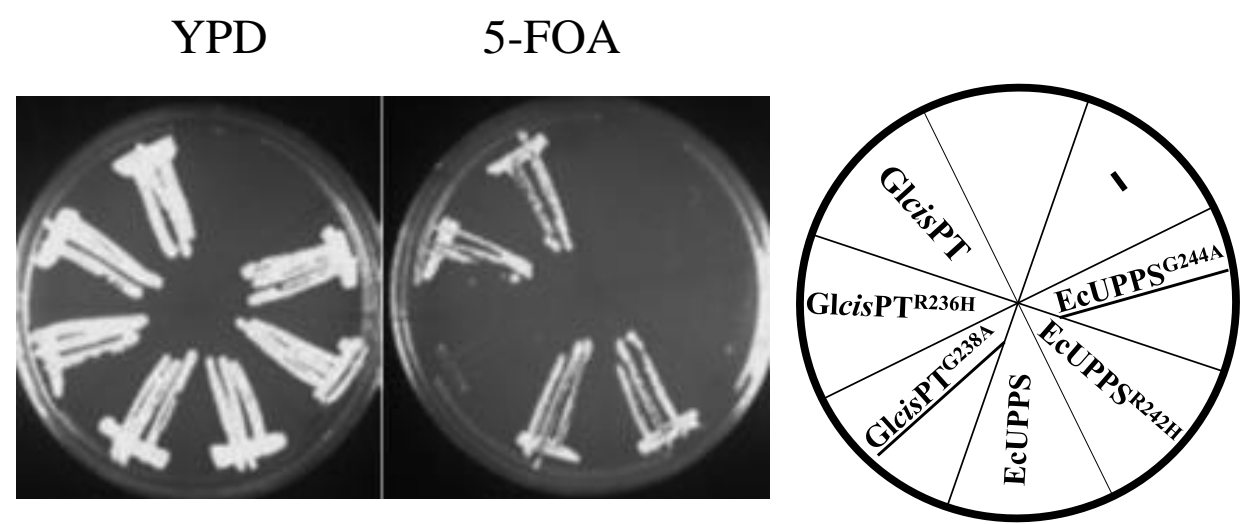

C.

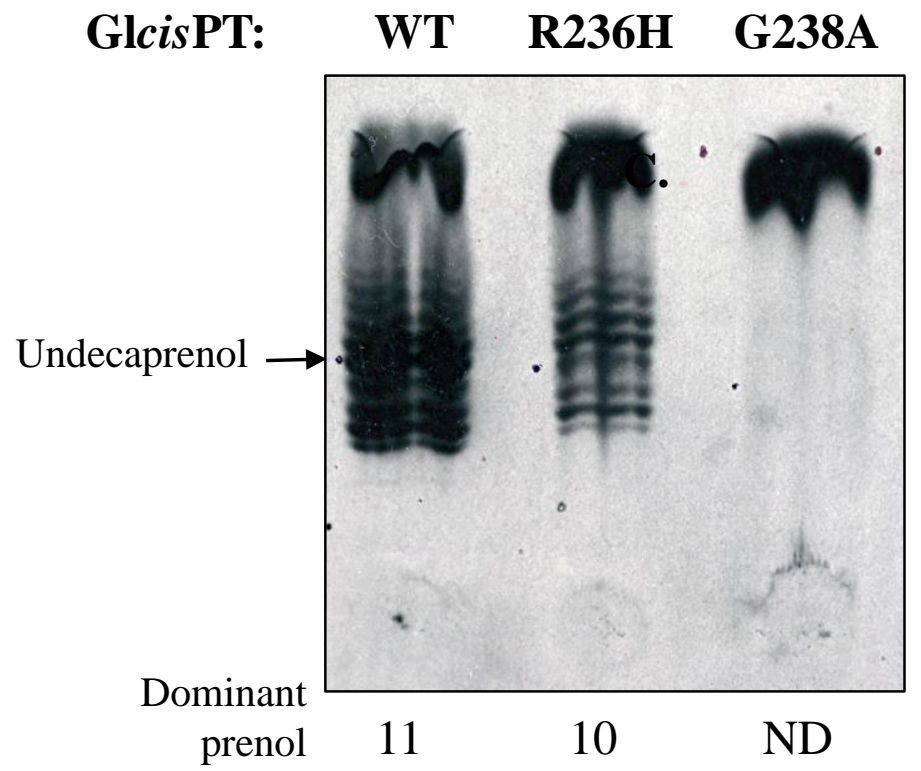

B.

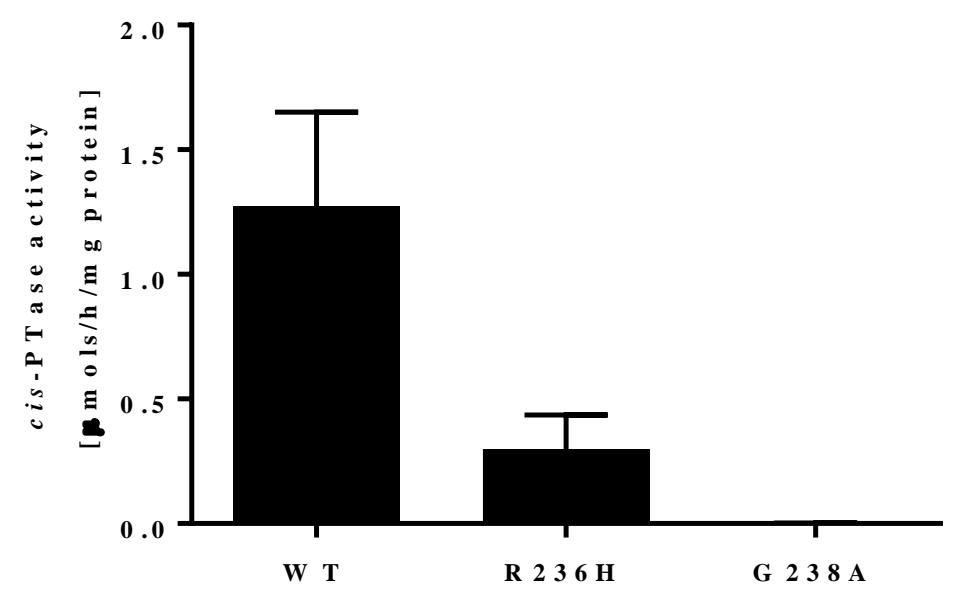




\section{Fig 6.}

A.

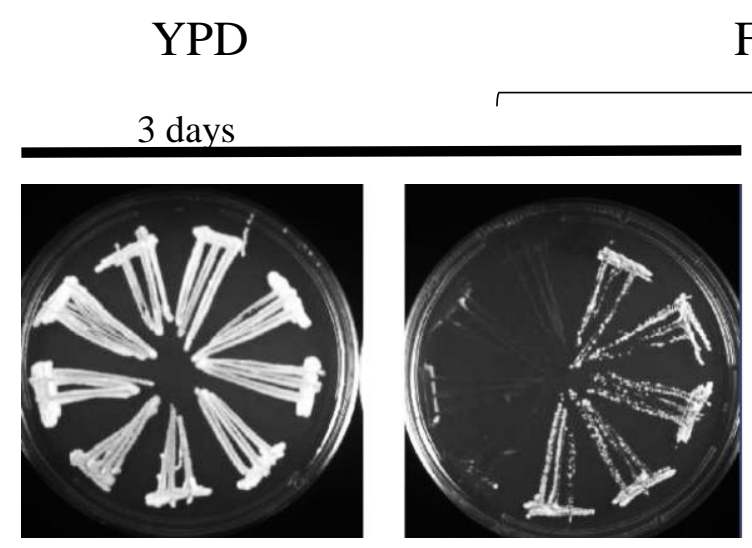

FOA
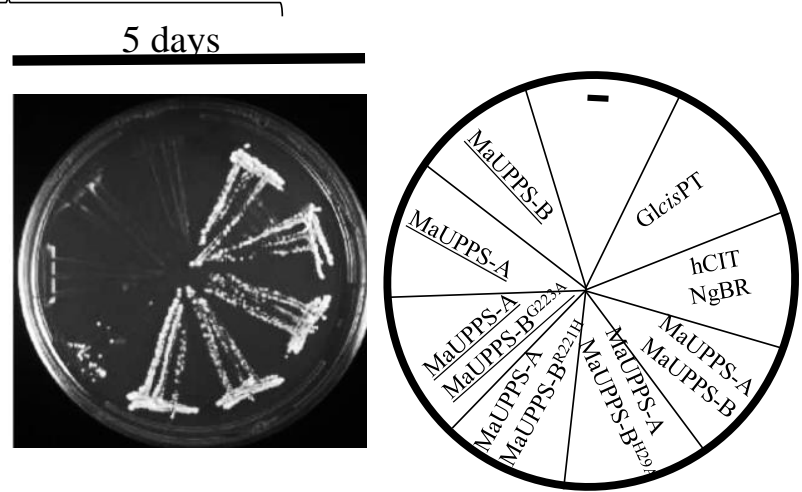

B.

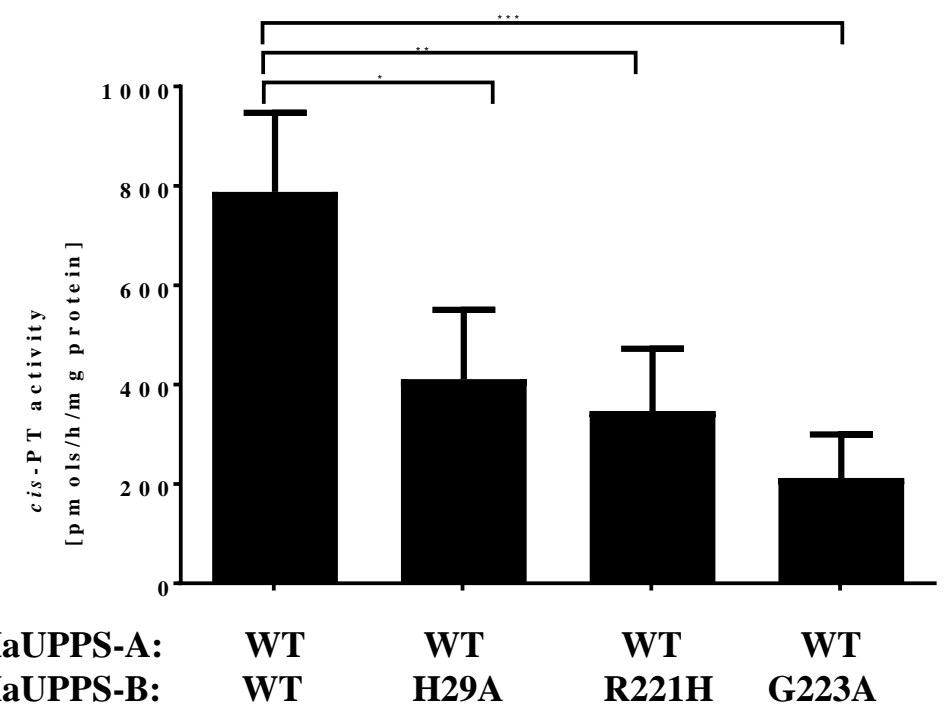

C.

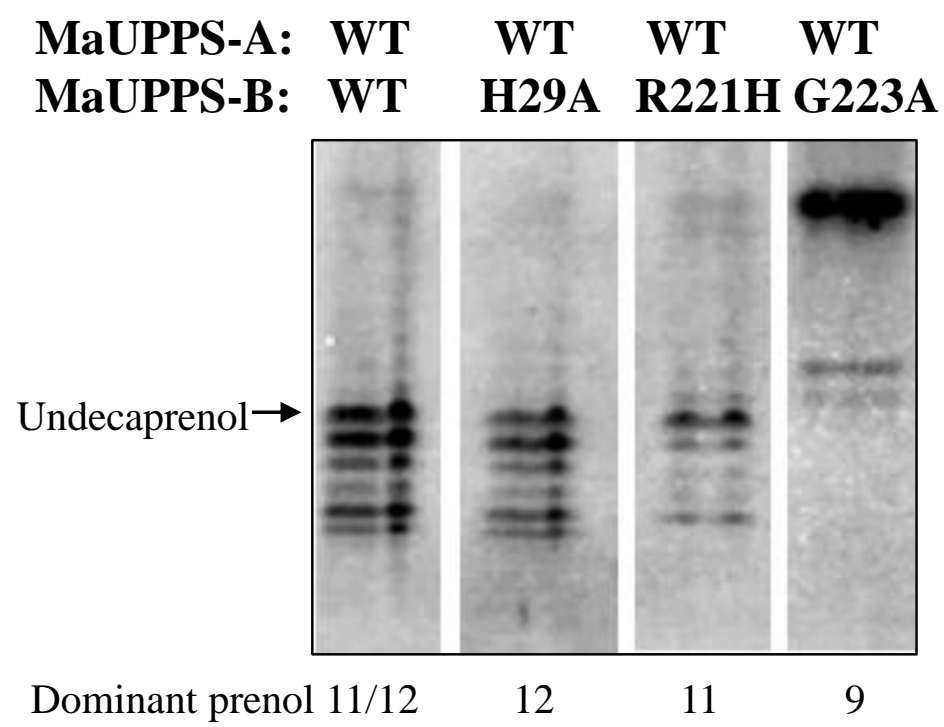

